\title{
Fuzzy-Model-Based Robust Control of Markov Jump Nonlinear Systems With Incomplete Transition Probabilities and Uncertain Packet Dropouts
}

\section{Zeyuan Xu}

Harbin Institute of Technology

Meng Joo Er ( $\nabla$ mjer@dlmu.edu.cn )

Dalian Maritime University https://orcid.org/0000-0003-4597-7088

\section{Research Article}

Keywords: Markov jump systems, incomplete transition probabilities, $\mathrm{H} \infty$ control, IT2 fuzzy systems, uncertain packet dropouts

Posted Date: June 11th, 2021

DOI: https://doi.org/10.21203/rs.3.rs-575881/v1

License: (c) (1) This work is licensed under a Creative Commons Attribution 4.0 International License. Read Full License 


\title{
Fuzzy-Model-Based Robust Control of Markov Jump Nonlinear Systems With Incomplete Transition Probabilities and Uncertain Packet Dropouts
}

\author{
Zeyuan Xu • Meng Joo Er
}

Received: date / Accepted: date

\begin{abstract}
Interval type-2 fuzzy Markov jump systems (IT2FMJSs) have received much attention because they can better describe complex nonlinear systems with uncertainties and stochastic system mode switching. Over the past decade, many excellent results of fuzzy MJSs (FMJSs) have been reported. However, the transition probabilities which govern the dynamic behaviour of MJSs have been assumed to be completely known, limiting real-world applications of existing results. Different from the previous studies, transition probabilities between system modes switching are partly unknown, and packet dropouts of data transmission are uncertain in this study. The main contributions of this work are: (1) To analyze stochastic stability and reduce conservatism, a novel Lyapunov function which both depends on system mode and fuzzy basis function is constructed; (2) The existence of a mode-dependent and fuzzy-basis-dependent state-feedback controller is investigated; (3) The closedloop system is stochastically stable with a desired $H_{\infty}$ performance, thereby addressing the problem of incomplete transition probabilities and uncertain packet dropouts. An illustrative example of a robot arm is used to demonstrate the effectiveness and practicality of the proposed approach. By virtue of the proposed approach, the effects of incomplete transition probabilities and uncertain packet dropouts on IT2FMJSs are alleviated.
\end{abstract}

Keywords Markov jump systems - incomplete transition probabilities $\cdot H_{\infty}$ control · IT2 fuzzy systems · uncertain packet dropouts

Zeyuan $\mathrm{Xu}$

Department of Control Science and Engineering, Harbin Institute of Technology, Harbin, 150001, China

Tel.: +86-157-65587282

E-mail: xuzeyuan@hit.edu.cn

Meng Joo Er

School of Marine Electrical Engineering, Dalian Maritime University, Dalian, 116026, China E-mail: mjer22690@gmail.com. 


\section{Introduction}

In practical engineering systems, most physical plants contain nonlinearities. Because of the capability of approximating nonlinear systems, Takagi-Sugeno (T-S) fuzzy models have been widely used in control systems [6,10,25]. Generally speaking, by blending all linear subsystems through a set of IF-THEN rules, the T-S fuzzy model can describe any smooth nonlinear system [25]. Up to now, works on fuzzy systems have been active, and many fruitful results on stability analysis, control synthesis, and filtering synthesis have been reported $[3,11,23,29,30]$. By considering nonlinear terms and uncertain parameters, the interval type-2 (IT2) technique has been introduced into T-S fuzzy systems to improve their approximation capabilities. By considering uncertain information and upper and lower membership functions (ULMFs), a stable IT2 fuzzy controller has been designed to control a process. Some typical control methods based on IT2 fuzzy systems (IT2FSs) have been proposed $[6,12]$. Moreover, studies on fuzzy systems have been witnessed in other fields such as sensor networks [16], switched systems [9,18], and multi-agent systems [36]. These latest research results make hybrid systems a mainstream trend due to the complexity of dynamic systems. However, many complex dynamic systems are not sufficiently expressed by IT2FSs, especially systems with stochastically varying parameters subject to the Markov process. In this context, IT2 fuzzy Markov jump systems (IT2FMJSs) can be adopted.

As a branch of hybrid systems, IT2FMJSs have received much attention because they can not only represent systems with Markovian stochastic variations such as stochastic characteristics of dynamic systems themselves, random failures or repairs of components, and sudden environmental disturbances, but also address nonlinearity and uncertainty of complex systems [35]. As such, IT2FMJSs can combine the advantages of IT2FSs and Markov jump systems (MJSs), which can better describe complex nonlinear systems with uncertainties and system mode switching. Over the past decade, many favorable results of fuzzy MJSs (FMJSs) have been reported, including stability $[8,28]$, stabilization [17], $H_{\infty}$ control [22,34], and filtering [21]. However, transition probabilities which govern the dynamic behavior of MJSs have been usually assumed to be completely known, which, to a great extent, limits wide spread applications of the research results because it is difficult to obtain complete description of transition probability $[31,32]$. In practical systems, MJSs with incomplete transition probability are more common and general than those with completely known transition probability. This has more necessary research significance from the control perspective. Many practical systems with unpredictable structural variations can be described by MJSs with generally incomplete transition information [19]. In [13], the $H_{\infty}$ control problem of MJSs considering time-varying delay and incomplete transition probability has been addressed. Furthermore, when sensor failures are considered, a dissipative filter of FMJSs with incomplete transition probability is proposed in [20]. Moreover, in $[4,15,26,27]$, other control and filtering methods of FMJSs with 
complete transition probability have been presented, but these researches rarely involved the control problem of incomplete transition probability.

In addition, because FMJSs is one kind of networked control systems (NC$\mathrm{Ss}$ ) in this paper, it is required to study the problem of data packet dropout in NCSs. In recent years, some solution methods to packet dropouts have been presented $[5,7,14,24,33]$. In $[7,33]$, a random process was adopted to describe the packet dropout process, among which the random process has completely or partly known transition probability. By modeling the packet dropout process using random variables that satisfy the Bernoulli distribution, the $H_{\infty}$ fuzzy control problem of FMJSs has been addressed in [2, 7]. However, almos all the aforementioned research results assume that the expectation of packet dropouts is certain. On the contrary, the expectation of packet dropout$\mathrm{s}$ is uncertain in practical systems. Regarding the aforementioned issues, few attempts have been made on the control problem of FMJSs with uncertain packet dropout.

Different from existing approaches, this paper focuses on controller de-sign of IT2FMJSs with incomplete transition probabilities and uncertain pack-et dropouts. The main contributions are summarized below.

1) IT2FMJSs with incomplete transition probabilities are more general than existing FMJSs, which addresses the difficulty to obtain the complete description of transition probability such that IT2FMJSs can be effectively controlled.

2) By considering that packet dropouts are uncertain and the transition probability is partly unknown, a mode-dependent state control strategy is proposed to simultaneously address the two problems.

3) Based on a novel fuzzy-basis-dependent and mode-dependent Lyapunov function, the obtained sufficient conditions guarantee that the resulting closed-loop systems are stochastically stable with the desired $H_{\infty}$ performance, which reduces conservatism.

Notation: $P>\mathbf{0}(P<\mathbf{0})$ indicates that $P$ is a positive (negative) definite matrix. $\emptyset$ denotes the empty set. In symmetric block matrices, the asterisk "*" denotes an ellipsis for the parts introduced by symmetry. $I_{n}$ and $\mathbf{0}$ are $n \times n$ identity matrix and zero matrix with appropriate dimension, respectively. $\operatorname{diag}\{\ldots\}$ denotes a block-diagonal matrix. $\operatorname{Pr}\{A \mid B\}$ denotes the condition probability of event $A$ conditional on $B . E\{a\}$ and $E\{a \mid b\}$ denote expectation of $a$ and expectation of $a$ conditional on $b$, respectively. Moreover, $\|\cdot\|_{E 2}$ denotes expected $l_{2}[0, \infty)$ norm.

\section{Preliminaries and model formulation}

\subsection{Physical Plant}

By considering the discrete-time IT2FMJS with incomplete transition probabilities and uncertain packet dropouts, the IT2FS is expressed as follows: 
Plant Rule $i$ : IF $f_{1, c_{k}}(k)$ is $\mathbb{F}_{1, c_{k}}^{i}, f_{2, c_{k}}(k)$ is $\mathbb{F}_{2, c_{k}}^{i}, \ldots$, and $f_{\beta, c_{k}}(k)$ is $\mathbb{F}_{\beta, c_{k}}^{i}$, THEN

$$
\left\{\begin{array}{l}
x(k+1)=A_{i, c_{k}} x(k)+B_{i, c_{k}} u(k)+G_{i, c_{k}} \xi(k) \\
z(k)=C_{i, c_{k}} x(k)+D_{i, c_{k}} u(k)+H_{i, c_{k}} \xi(k)
\end{array}\right.
$$

where $i \in R \triangleq\{1,2, \ldots, r\}$, and $r$ is the number of fuzzy rules; $\left\{c_{k}, k \geq 0\right\}$ denotes a discrete-time Markov chain, and $c_{k} \in M \triangleq\{1,2, \ldots, \alpha\} ; f_{c_{k}}(k)=$ $\left\{f_{1, c_{k}}(k), \ldots, f_{\beta, c_{k}}(k)\right\}$ is the premise variable vector, and $\beta$ is the number of premise variables; $\mathbb{F}_{a, c_{k}}^{i}\left(i \in R, a \in\{1,2, \ldots, \beta\}\right.$, and $\left.c_{k} \in M\right)$ is the fuzzy set; $x(k) \in \mathbb{R}^{n}$ denotes the system state, $u(k) \in \mathbb{R}^{m}$ represents the control input, $z(k) \in \mathbb{R}^{t}$ stands for the control output, and $\xi(k) \in \mathbb{R}^{s}$ denotes the disturbance input; $A_{i, c_{k}}, B_{i, c_{k}}, C_{i, c_{k}}, D_{i, c_{k}}, G_{i, c_{k}}$, and $H_{i, c_{k}}$ are known real-valued matrix functions with respect to $c_{k}$. The firing interval of the $i$ th rule can be depicted by

$$
\mathfrak{M}_{i}\left(f_{c_{k}}(k)\right) \in\left[\underline{\lambda}_{i}\left(f_{c_{k}}(k)\right), \bar{\lambda}_{i}\left(f_{c_{k}}(k)\right)\right]
$$

where $\underline{\lambda}_{i}\left(f_{c_{k}}(k)\right)$ and $\bar{\lambda}_{i}\left(f_{c_{k}}(k)\right)$ denote the lower and upper fuzzy basis functions (LUFBFs) for the $i$ th rule,

$$
\begin{aligned}
\underline{\lambda}_{i}\left(f_{c_{k}}(k)\right) & =\frac{\underline{\omega}_{i}\left(f_{c_{k}}(k)\right)}{\sum_{i=1}^{r} \underline{\omega}_{i}\left(f_{c_{k}}(k)\right)} \\
\underline{\omega}_{i}\left(f_{c_{k}}(k)\right) & =\prod_{a=1}^{\beta} \underline{\mathbb{F}}_{a, c_{k}}^{i}\left(f_{a, c_{k}}(k)\right) \\
\bar{\lambda}_{i}\left(f_{c_{k}}(k)\right) & =\frac{\bar{\omega}_{i}\left(f_{c_{k}}(k)\right)}{\sum_{i=1}^{r} \bar{\omega}_{i}\left(f_{c_{k}}(k)\right)} \\
\bar{\omega}_{i}\left(f_{c_{k}}(k)\right) & =\prod_{a=1}^{\beta} \overline{\mathbb{F}}_{a, c_{k}}^{i}\left(f_{a, c_{k}}(k)\right)
\end{aligned}
$$

and $\mathbb{F}_{a, c_{k}}^{i}\left(f_{a, c_{k}}(k)\right)$ is the grade of membership of $f_{a, c_{k}}(k)$ in $\mathbb{F}_{a, c_{k}}^{i}$. Hence, the fuzzy basis function is

$$
\begin{aligned}
& \lambda_{i}\left(f_{c_{k}}(k)\right)=\underline{\mu}_{i}\left(f_{c_{k}}(k)\right) \underline{\lambda}_{i}\left(f_{c_{k}}(k)\right)+\bar{\mu}_{i}\left(f_{c_{k}}(k)\right) \bar{\lambda}_{i}\left(f_{c_{k}}(k)\right) \\
& \sum_{i=1}^{r} \lambda_{i}\left(f_{c_{k}}(k)\right)=1, \underline{\mu}_{i}\left(f_{c_{k}}(k)\right)+\bar{\mu}_{i}\left(f_{c_{k}}(k)\right)=1
\end{aligned}
$$

where $\lambda_{i}\left(f_{c_{k}}(k)\right)$ denotes fuzzy basis functions for the $i$ th rule, $\mu_{i}\left(f_{c_{k}}(k)\right)$ and $\bar{\mu}_{i}\left(f_{c_{k}}(k)\right)$ are the lower and upper weighting coefficient functions (LUWCFs) that can capture variations of uncertain parameters. In the sequel, $\lambda_{i}\left(f_{c_{k}}(k)\right)$ is denoted by $\lambda_{i, c_{k}}$ for simplicity.

By leveraging strategies of singleton fuzzification, product inference, and center-average defuzzification, the global model of discrete-time IT2FMJS can 
be written as

$$
\left\{\begin{array}{l}
x(k+1)=\sum_{i=1}^{r} \lambda_{i, c_{k}}\left\{A_{i, c_{k}} x(k)+B_{i, c_{k}} u(k)+G_{i, c_{k}} \xi(k)\right\} \\
z(k)=\sum_{i=1}^{r} \lambda_{i, c_{k}}\left\{C_{i, c_{k}} x(k)+D_{i, c_{k}} u(k)+H_{i, c_{k}} \xi(k)\right\}
\end{array}\right.
$$

\subsection{Partly Unknown Transition Probability}

The stochastic process $\left\{c_{k}, k \geq 0\right\}$ is modelled by a discrete-time Markov chain, the transition probability between mode switching is given by

$$
\operatorname{Pr}\left(c_{k+1}=n \mid c_{k}=m\right)=\rho_{m n}
$$

where $\forall m, n \in M, \rho_{m n} \geq 0$, and $\sum_{n=1}^{\alpha} \rho_{m n}=1$. Moreover, define the transition probability matrix (TPM) as

$$
\Upsilon=\left[\begin{array}{cccc}
\rho_{11} & ? & \cdots & \rho_{1 \alpha} \\
? & ? & \cdots & \rho_{2 \alpha} \\
\vdots & \vdots & \ddots & \vdots \\
? & ? & \cdots & \rho_{\alpha \alpha}
\end{array}\right]
$$

where "?" denotes inaccessible elements. For the convenience of analysis, $\forall m \in$ $M, M^{(m)}=M_{K}^{(m)}+M_{U K}^{(m)}$ is denoted by $M_{K}^{(m)} \triangleq\left\{n: \rho_{m n}\right.$ is known $\}$ and $M_{U K}^{(m)} \triangleq\left\{n: \hat{\rho}_{m n}\right.$ is unknown $\}$. Furthermore, if $M_{K}^{(m)} \neq \emptyset, M_{K}^{(m)}$ can be also described as $M_{K}^{(m)}=\left\{K_{1}^{(m)}, K_{2}^{(m)}, \ldots, K_{b}^{(m)}\right\}, \forall 1 \leqslant b \leqslant \alpha$, where $K_{b}^{(m)} \in \mathbb{N}^{+}$ denotes the $b$ th known element in the $m$ th row of the matrix $\Upsilon$. Besides, sum of known elements and sum of unknown elements, respectively, are denoted by

$$
\rho_{K}^{(m)} \triangleq \sum_{n \in M_{K}^{(m)}} \rho_{m n}, \rho_{U K}^{(m)} \triangleq \sum_{n \in M_{U K}^{(m)}} \hat{\rho}_{m n}
$$

\subsection{Uncertain Packet Dropout}

Since the packet dropout occurs in communication links between sensors and the controller as well as between the controller and the actuator, a random process is adopted to describe the packet dropout, as follows:

$$
x_{d}(k)=\chi(k) x(k), u(k)=\delta(k) u_{d}(k)
$$

where $x_{d}(k)$ and $u_{d}(k)$ are actual input and output of the controller due to the packet dropout, respectively. The terms $\chi(k)$ and $\delta(k)$ are individually independent Bernoulli processes. In other words, $\chi(k)=1$ (or $\chi(k)=0$ ) when data transmission of the links succeeds (or fails). Similarly, $\delta(k)$ also holds. By defining

$$
\varepsilon(k) \triangleq \chi(k) \delta(k)
$$


two independent processes are combined into one. If $\chi(k)=\delta(k)=1, \varepsilon(k)=1$, and $\varepsilon(k)=0$, otherwise. Moreover, by considering uncertain packet dropout, the following is obtained:

$$
\left\{\begin{array}{l}
\operatorname{Pr}\{\varepsilon(k)=1\}=\bar{\varepsilon}+\Delta \varepsilon, \operatorname{Pr}\{\varepsilon(k)=0\}=1-\bar{\varepsilon}-\Delta \varepsilon \\
E\{\varepsilon(k)\}=E\left\{\varepsilon^{2}(k)\right\}=\bar{\varepsilon}+\Delta \varepsilon
\end{array}\right.
$$

where $\bar{\varepsilon}+\Delta \varepsilon$ denotes the success packet rate, and $\bar{\varepsilon}$ and $\Delta \varepsilon$ are the nominal expectation and norm-bounded uncertainty of the success packet rate, respectively. By defining $-\sigma \leq \Delta \varepsilon \leq \sigma$, we have $\bar{\varepsilon}+\Delta \varepsilon(k) \leqslant \bar{\varepsilon}+\sigma, 1-\bar{\varepsilon}-\Delta \varepsilon(k) \leqslant$ $1-\bar{\varepsilon}+\sigma$. Combining (9) and (10) yields

$$
u(k)=\sum_{j=1}^{r} \lambda_{j, c_{k}} K_{j, c_{k}} \varepsilon(k) x(k)
$$

\subsection{Closed-loop System}

By substituting (12) into (5), the resulting closed-loop system is written as

$$
\left\{\begin{array}{l}
x(k+1)=\sum_{i=1}^{r} \sum_{j=1}^{r} \lambda_{i, c_{k}} \lambda_{j, c_{k}}\left\{\bar{A}_{i j, c_{k}} x(k)+G_{i, c_{k}} \xi(k)\right\} \\
z(k)=\sum_{i=1}^{r} \sum_{j=1}^{r} \lambda_{i, c_{k}} \lambda_{j, c_{k}}\left\{\bar{C}_{i j, c_{k}} x(k)+H_{i, c_{k}} \xi(k)\right\}
\end{array}\right.
$$

where $\bar{A}_{i j, c_{k}}=A_{i, c_{k}}+\varepsilon(k) B_{i, c_{k}} K_{j, c_{k}}$ and $\bar{C}_{i j, c_{k}}=C_{i, c_{k}}+\varepsilon(k) D_{i, c_{k}} K_{j, c_{k}}$.

Before proceeding, the following definition about stability and performance of IT2FMJSs is introduced.

Definition 1 (see [1]) The closed-loop system (13) with $\xi(k) \equiv 0(k=$ $0,1, \ldots)$ is stochastically stable in the mean square sense, if any initial condition $x(0) \in \mathbb{R}^{n}$ and $c_{0} \in M$ satisfy

$$
E\left\{\sum_{k=0}^{\infty}|x(k)|^{2} \mid x(0), c_{0}\right\}<\infty
$$

For the system (5), the TPM is incomplete and the packet dropout is uncertain. By addressing the above two problems, a fuzzy $H_{\infty}$ controller with a prescribed scalar $\gamma>0$ in the form of (12) is designed for any $\rho_{m n}$ satisfying (8).

(1) In the sense of (14), the closed-loop IT2FMJS given by (13) is stochastically stable.

(2) The control output $z(k)$ satisfies $\|z(k)\|_{E 2} \leqslant \gamma\|\xi(k)\|_{E 2}$ with zero initial condition, where $\|z(k)\|_{E 2} \triangleq E\left\{\sum_{k=0}^{\infty}\left[z^{T}(k) z(k)\right]\right\}^{\frac{1}{2}}$. 


\section{Main results}

3.1 Stochastic Stability Analysis

The $H_{\infty}$ performance problem for the closed-loop system (13) will be addressed. Sufficient conditions will be obtained such that the closed-loop system (13) is stochastically stable with the optimized $H_{\infty}$ performance index.

Theorem 1 By considering incomplete transition probabilities and uncertain packet dropouts, for a guaranteed $H_{\infty}$ performance index $\gamma>0, \bar{\varepsilon}>0, \Delta \varepsilon>$ $0, \sigma>0$, the discrete-time IT2FMJS (13) is stochastically stable for any uncertain packet dropout rate satisfying $-\sigma \leq \Delta \varepsilon \leq \sigma$, if there exist positive definite matrices $P_{i, m}, i \in R, m \in M$, and symmetric matrices $\Psi_{i j l, m}, i, j, l \in$ $R, m \in M$, such that

$$
\left\{\begin{array}{l}
\mathcal{A}_{i j, m}^{T} \mathcal{B A}_{i j, m}-\mathcal{C}_{i, m}+\Psi_{i j l, m}<0 \\
\Psi_{l, m}>0,(i, j, l \in R, i \geqslant j, m, n \in M)
\end{array}\right.
$$

where

$$
\begin{aligned}
& \mathcal{A}_{i j, m}=\left[\begin{array}{cc}
\Phi_{i j, m} & \Theta_{i j, m} \\
\Omega_{i j, m} & \Xi_{i j, m}
\end{array}\right], \mathcal{C}_{i, m}=\frac{1}{r}\left[\begin{array}{cc}
P_{i, m} & * \\
0 & \gamma^{2} I
\end{array}\right] \\
& \mathcal{B}=\left[\begin{array}{cc}
\widehat{P} & * \\
0 & r \widehat{I}
\end{array}\right], \Psi_{i j l, m}=\left[\begin{array}{cc}
U_{i j l, m} & * \\
V_{i j l, m} & W_{i j l, m}
\end{array}\right] \\
& \Psi_{l, m}=\left[\begin{array}{cccc}
\Psi_{11 l, m} & * & \cdots & * \\
\Psi_{21 l, m} & \Psi_{22 l, m} & \cdots & * \\
\vdots & \vdots & \ddots & \vdots \\
\Psi_{r 1 l, m} & \Psi_{r 2 l, m} & \cdots & \Psi_{r r l, m}
\end{array}\right] \\
& \Phi_{i j, m}=\left[\begin{array}{l}
\theta_{1} \mathcal{D}_{i j, m} \\
\theta_{2} S_{i j, m}
\end{array}\right], \Omega_{i j, m}=\left[\begin{array}{l}
\theta_{1}\left(C_{i, m}+C_{j, m}\right) / 2 \\
\theta_{2} T_{i j, m}
\end{array}\right] \\
& \Theta_{i j, m}=\left[\begin{array}{c}
\theta_{1} \mathcal{E}_{i j, m} \\
\theta_{2} \mathcal{E}_{i j, m}
\end{array}\right], \Xi_{i j, m}=\left[\begin{array}{c}
\theta_{1}\left(H_{i, m}+H_{j, m}\right) / 2 \\
\theta_{2}\left(H_{i, m}+H_{j, m}\right) / 2
\end{array}\right] \\
& \widehat{P}=\left[\begin{array}{ll}
P & 0 \\
0 & P
\end{array}\right], \widehat{I}=\left[\begin{array}{ll}
I & 0 \\
0 & I
\end{array}\right] \\
& \mathcal{D}_{i j, m}=\frac{1}{2}\left(A_{i, m}+A_{j, m}\right), \quad \mathcal{E}_{i j, m}=\frac{1}{2}\left(G_{i, m}+G_{j, m}\right) \\
& \theta_{1}=\sqrt{1-\bar{\varepsilon}+\sigma}, \theta_{2}=\sqrt{\bar{\varepsilon}+\sigma} \\
& S_{i j, m}=\frac{1}{2}\left(A_{i, m}+A_{j, m}+B_{i, m} K_{j, m}+B_{j, m} K_{i, m}\right) \\
& T_{i j, m}=\frac{1}{2}\left(C_{i, m}+C_{j, m}+D_{i, m} K_{j, m}+D_{j, m} K_{i, m}\right) \\
& P=\sum_{n \in M_{K}^{(m)}} \rho_{m n} P_{l, n}+\left(1-\rho_{K}^{(m)}\right) P_{l, v}
\end{aligned}
$$


Proof By defining $c_{k}=m, c_{k+1}=n$, the following mode-dependent and fuzzybasis-dependent Lyapunov function is constructed:

$$
V\left(k, c_{k}\right)=x^{T}(k)\left(\sum_{i=1}^{r} \lambda_{i, c_{k}} P_{i, c_{k}}\right) x(k)
$$

Stochastic stability of the closed-loop system (13) without disturbance input, i.e., $\xi(k) \equiv 0(k=1,2, \ldots)$, is now analyzed.

By defining $\bar{P}_{l, n}=\sum_{l=1}^{r} \sum_{n \in M^{(m)}} \rho_{m n} \lambda_{l, n} P_{l, n}=\sum_{l=1}^{r} \lambda_{l, n} P$, the expectation of any increment of $V(k)$ is given by

$$
\begin{aligned}
E\{ & \Delta V(k)\} \\
= & x^{T}(k) E\left\{\sum_{i=1}^{r} \sum_{j=1}^{r} \sum_{g=1}^{r} \sum_{h=1}^{r} \lambda_{i, m} \lambda_{j, m} \lambda_{g, m} \lambda_{h, m}\right. \\
& \left.\times \bar{A}_{i j, m}^{T} \bar{P}_{l, n} \bar{A}_{g h, m}-\sum_{i=1}^{r} \lambda_{i, m} P_{i, m}\right\} x(k) \\
= & x^{T}(k) E\left\{\sum_{i=1}^{r} \sum_{j=1}^{r} \sum_{g=1}^{r} \sum_{h=1}^{r} \lambda_{i, m} \lambda_{j, m} \lambda_{g, m} \lambda_{h, m}\right. \\
& \times \frac{1}{4}\left(\bar{A}_{i j, m}^{T}+\bar{A}_{j i, m}^{T}\right) \bar{P}_{l, n}\left(\bar{A}_{g h, m}+\bar{A}_{h g, m}\right) \\
& \left.-\sum_{i=1}^{r} \lambda_{i, m} P_{i, m}\right\} x(k) \\
\leq & x^{T}(k) E\left\{\sum_{i=1}^{r} \sum_{j=1}^{r} \sum_{g=1}^{r} \sum_{h=1}^{r} \lambda_{i, m} \lambda_{j, m} \lambda_{g, m} \lambda_{h, m}\right. \\
& \times \frac{1}{8}\left[\left(\bar{A}_{i j, m}+\bar{A}_{j i, m}\right)^{T} \bar{P}_{l, n}\left(\bar{A}_{i j, m}+\bar{A}_{j i, m}\right)\right. \\
& \left.+\left(\bar{A}_{g h, m}+\bar{A}_{h g, m}\right)^{T} \bar{P}_{l, n}\left(\bar{A}_{g h, m}+\bar{A}_{h g, m}\right)\right] \\
& \left.-\sum_{i=1}^{r} \lambda_{i, m} P_{i, m}\right\} x(k) \\
& \left.\times \bar{P}_{l, n}\left(\bar{A}_{i j, m}+\bar{A}_{j i, m}\right)-\sum_{i=1}^{r} \lambda_{i, m} P_{i, m}\right\} x(k) \\
& \sum_{i=1}^{r} \sum_{j=1}^{r} \frac{1}{4} \lambda_{i, m} \lambda_{j, m}\left(\bar{A}_{i j, m}+\bar{A}_{j i, m}\right)^{T} \\
& \\
& \\
&
\end{aligned}
$$


Following the above derivation, (18) can be written as

$$
\begin{aligned}
E\{ & \Delta V(k)\} \\
\leq & x^{T}(k) E\left\{\sum_{i=1}^{r} \sum_{j=1}^{r} \lambda_{i, m} \lambda_{j, m} \overline{\mathcal{D}}_{i j, m}^{T} \bar{P}_{l, n} \overline{\mathcal{D}}_{i j, m}\right. \\
& \left.-\sum_{i=1}^{r} \lambda_{i, m} P_{i, m}\right\} x(k) \\
\leq & x^{T}(k) \sum_{i=1}^{r} \sum_{j=1}^{r} \lambda_{i, m} \lambda_{j, m}\left\{(1-\bar{\varepsilon}-\Delta \varepsilon) \mathcal{D}_{i j, m}^{T} \bar{P}_{l, n} \mathcal{D}_{i j, m}\right. \\
& \left.+(\bar{\varepsilon}+\Delta \varepsilon) S_{i j, m}^{T} \bar{P}_{l, n} S_{i j, m}-P_{i, m}\right\} x(k) \\
< & x^{T}(k) \sum_{l=1}^{r} \sum_{i=1}^{r} \sum_{j=1}^{r} \lambda_{i, m} \lambda_{j, m} \lambda_{l, n}\left\{\theta_{1}^{2} \mathcal{D}_{i j, m}^{T} P \mathcal{D}_{i j, m}\right. \\
& \left.+\theta_{2}^{2} S_{i j, m}^{T} P S_{i j, m}-\frac{1}{r} P_{i, m}\right\} x(k) \\
< & x^{T}(k) \sum_{l=1}^{r} \sum_{i=1}^{r} \sum_{j=1}^{r} \lambda_{i, m} \lambda_{j, m} \lambda_{l, n}\left\{\Phi_{i j, m}^{T} \widehat{P} \Phi_{i j, m}\right. \\
& \left.-\frac{1}{r} P_{i, m}\right\} x(k) \\
< & x^{T}(k) \sum_{l=1}^{r} \sum_{i=1}^{r} \lambda_{i, m}^{2} \lambda_{l, n}\left\{\Phi_{i i, m}^{T} \widehat{P} \Phi_{i i, m}-\frac{1}{r} P_{i, m}\right\} x(k) \\
& +2 x^{T}(k) \sum_{l=1}^{r} \sum_{i=1}^{r} \sum_{i>j}^{r} \lambda_{i, m} \lambda_{j, m} \lambda_{l, n}\left\{\Phi_{i j, m}^{T} \widehat{P} \Phi_{i j, m}\right. \\
& \left.-\frac{1}{r} P_{i, m}\right\} x(k)
\end{aligned}
$$

where

$$
\begin{aligned}
& \overline{\mathcal{D}}_{i j, m}=\frac{1}{2}\left(\bar{A}_{i j, m}+\bar{A}_{j i, m}\right), \mathcal{D}_{i j, m}=\frac{1}{2}\left(A_{i, m}+A_{j, m}\right) \\
& \bar{A}_{i j, m}=A_{i, m}+\varepsilon(k) B_{i, m} K_{j, m}, \widehat{P}=\left[\begin{array}{cc}
P & 0 \\
0 & P
\end{array}\right] \\
& \bar{A}_{j i, m}=A_{j, m}+\varepsilon(k) B_{j, m} K_{i, m}, \Phi_{i i, m}=\left[\begin{array}{l}
\theta_{1} A_{i, m} \\
\theta_{2} S_{i i, m}
\end{array}\right] \\
& S_{i i, m}=A_{i, m}+B_{i, m} K_{i, m}
\end{aligned}
$$

From (15), the following are obtained:

$$
\left\{\begin{array}{l}
\Phi_{i i, m}^{T} \widehat{P} \Phi_{i i, m}+r \Omega_{i i, m}^{T} \Omega_{i i, m}-\frac{1}{r} P_{i, m}+U_{i i l, m} \\
<0, \quad(i=j) \\
\Phi_{i j, m}^{T} \widehat{P} \Phi_{i j, m}+r \Omega_{i j, m}^{T} \Omega_{i j, m}-\frac{1}{r} P_{i, m}+U_{i j l, m} \\
<0, \quad(i>j)
\end{array}\right.
$$


Thus, it can be seen from (20) that

$$
\Phi_{i j, m}^{T} \widehat{P} \Phi_{i j, m}-\frac{1}{r} P_{i, m}+U_{i j l, m}<0,(i \geqslant j)
$$

Therefore, by combining (19) with (21), it is easy to obtain

$$
\begin{aligned}
E\{\Delta V(k)\} & <-\sum_{l=1}^{r} \sum_{i=1}^{r} \sum_{j=1}^{r} \lambda_{l, n} \lambda_{i, m} \lambda_{j, m} x^{T}(k) U_{i j l, m} x(k) \\
& =-\sum_{l=1}^{r} \lambda_{l, n} x^{T}(k) \Lambda^{T} \aleph \Lambda x(k)
\end{aligned}
$$

where

$$
\begin{aligned}
\Lambda= & {\left[\begin{array}{cccc}
\lambda_{1, m} & \lambda_{2, m} & \cdots & \lambda_{r, m}
\end{array}\right]^{T} } \\
\aleph= & {\left[\begin{array}{cccc}
U_{11 l, m} & * & \cdots & * \\
U_{21 l, m} & U_{22 l, m} & \cdots & * \\
\vdots & \vdots & \ddots & \vdots \\
U_{r 1 l, m} & U_{r 2 l, m} & \cdots & U_{r r l, m}
\end{array}\right] }
\end{aligned}
$$

If $\Psi_{l, m}>0$ in (15) for any $l \in R, m \in M$, it is easy to obtain $\aleph>0$ for any $l \in R, m \in M$. Therefore, $E\{\Delta V(k)\}<0$, which proves that the IT2FMJS given by (13) with $\xi(k) \equiv 0$ is stochastically stable.

Next, the disturbance input $\xi(k)$ is introduced to in the system (13). Define

$$
J \triangleq E\left\{\sum_{k=0}^{\infty}\left[\Delta V(k)+z^{T}(k) z(k)-\gamma^{2} \xi^{T}(k) \xi(k)\right]\right\}
$$


When $\xi(k)$ is considered, by defining $\psi_{k}=\left[x_{k}^{T} \xi_{k}^{T}\right]^{T}$, the expectation of $\Delta V(k)$ is obtained as follows:

$$
\begin{aligned}
E\{ & \Delta V(k)\} \\
= & E\left\{\sum_{i=1}^{r} \sum_{j=1}^{r} \sum_{p=1}^{r} \sum_{q=1}^{r} \lambda_{i, m} \lambda_{j, m} \lambda_{p, m} \lambda_{q, m}\right. \\
& \left.\times \overline{\mathcal{F}}_{i j, m}^{T} \bar{P}_{l, n} \overline{\mathcal{F}}_{p q, m}-x^{T}(k)\left(\sum_{i=1}^{r} h_{i, m} P_{i, m}\right) x(k)\right\} \\
< & \sum_{i=1}^{r} \sum_{j=1}^{r} \lambda_{i, m} \lambda_{j, m}\left\{\theta_{1}^{2} \mathcal{F}^{\prime}{ }_{i j, m} \bar{P}_{l, n} \mathcal{F}^{\prime}{ }_{i j, m}+\theta_{2}^{2} \mathcal{F}^{\prime \prime}{ }_{i j, m}\right. \\
& \left.\times \bar{P}_{l, n} \mathcal{F}^{\prime \prime}{ }_{i j, m}\right\}-x^{T}(k)\left\{\sum_{i=1}^{r} \lambda_{i, m} P_{i, m}\right\} x(k) \\
< & \psi^{T}(k) \sum_{i=1}^{r} \sum_{j=1}^{r} \lambda_{i, m} \lambda_{j, m}\left\{\mathcal{G}_{i j, m}^{T} \overline{\bar{P}}_{l, n} \mathcal{G}_{i j, m}\right\} \psi(k) \\
& -x^{T}(k)\left\{\sum_{i=1}^{r} \lambda_{i, m} P_{i, m}\right\} x(k)
\end{aligned}
$$

where

$$
\begin{aligned}
& \overline{\mathcal{F}}_{i j, m}=\overline{\mathcal{D}}_{i j, m} x(k)+\mathcal{E}_{i j, m} \xi(k) \\
& \overline{\mathcal{F}}_{p q, m}=\overline{\mathcal{D}}_{p q, m} x(k)+\mathcal{E}_{p q, m} \xi(k) \\
& \mathcal{F}^{\prime}{ }_{i j, m}=\mathcal{D}_{i j, m} x(k)+\mathcal{E}_{i j, m} \xi(k) \\
& \mathcal{F}^{\prime \prime}{ }_{i j, m}=S_{i j, m} x(k)+\mathcal{E}_{i j, m} \xi(k) \\
& \mathcal{G}_{i j, m}=\left[\Phi_{i j, m} \Theta_{i j, m}\right], \bar{P}_{l, n}=\left[\begin{array}{cc}
\bar{P}_{l, n} & 0 \\
0 & \bar{P}_{l, n}
\end{array}\right]
\end{aligned}
$$

Similarly, by referring to (13) and (24), we obtain

$$
\begin{aligned}
E & \left\{z^{T}(k) z(k)\right\} \\
\leq & \psi^{T}(k) \sum_{i=1}^{r} \sum_{j=1}^{r} \lambda_{i, m} \lambda_{j, m}\left\{\left[\Omega_{i j, m} \Xi_{i j, m}\right]^{T}\right. \\
& \left.\times\left[\Omega_{i j, m} \Xi_{i j, m}\right]\right\} \psi(k) \\
\leq & \psi^{T}(k) \sum_{i=1}^{r} \sum_{j=1}^{r} \lambda_{i, m} \lambda_{j, m}\left\{\mathcal{J}_{i j, m}^{T} \mathcal{J}_{i j, m}\right\} \psi(k)
\end{aligned}
$$

where $\mathcal{J}_{i j, m}=\left[\Omega_{i j, m} \Xi_{i j, m}\right]$. 
Therefore, combining (24) with (26) yields

$$
\begin{aligned}
E\{ & \left.\Delta V(k)+z^{T}(k) z(k)-\gamma^{2} \xi^{T}(k) \xi(k)\right\} \\
< & \psi^{T}(k) \sum_{i=1}^{r} \sum_{j=1}^{r} \lambda_{i, m} \lambda_{j, m}\left\{\mathcal{G}_{i j, m}^{T} \widehat{\bar{P}}_{l, n} \mathcal{G}_{i j, m}\right\} \psi(k) \\
& -x^{T}(k)\left(\sum_{i=1}^{r} \lambda_{i, m} P_{i, m}\right) x(k)-\gamma^{2} \xi^{T}(k) \xi(k) \\
& +\psi^{T}(k) \sum_{i=1}^{r} \sum_{j=1}^{r} \lambda_{i, m} \lambda_{j, m}\left\{\mathcal{J}_{i j, m}^{T} \mathcal{J}_{i j, m}\right\} \psi(k) \\
< & \psi^{T}(k) \sum_{i=1}^{r} \sum_{j=1}^{r} \lambda_{i, m} \lambda_{j, m}\left\{\sum_{l=1}^{r} \lambda_{l, n} \mathcal{G}_{i j, m}^{T} \widehat{P} \mathcal{G}_{i j, m}\right. \\
& \left.+\mathcal{J}_{i j, m}^{T} \mathcal{J}_{i j, m}-\mathcal{C}_{i, m}\right\} \psi(k) \\
< & \psi^{T}(k) \sum_{i=1}^{r} \sum_{j=1}^{r} \sum_{l=1}^{r} \lambda_{i, m} \lambda_{j, m} \lambda_{l, n}\left\{\mathcal{A}_{i j, m}^{T} \mathcal{B \mathcal { A }}_{i j, m}\right. \\
& \left.-\mathcal{C}_{i, m}\right\} \psi(k) \\
< & \psi^{T}(k) \sum_{i=1}^{r} \sum_{l=1}^{r} \lambda_{i, m}^{2} \lambda_{l, n}\left\{\mathcal{A}_{i i, m}^{T} \mathcal{B A}_{i i, m}-\mathcal{C}_{i, m}\right\} \psi(k) \\
& +2 \psi^{T}(k) \sum_{i=1}^{r} \sum_{i>j}^{r} \sum_{l=1}^{r} \lambda_{i, m} \lambda_{j, m} \lambda_{l, n}\left\{\mathcal{A}_{i j, m}^{T} \mathcal{B} \mathcal{A}_{i j, m}\right. \\
& \left.-\mathcal{C}_{i, m}\right\} \psi(k)
\end{aligned}
$$

where $\widehat{\bar{P}}_{l, n}=\sum_{l=1}^{r} \lambda_{l, n} \widehat{P}$.

Combining (15) and (27) yields

$$
\begin{aligned}
E & \left\{\Delta V(k)+z^{T}(k) z(k)-\gamma^{2} \xi^{T}(k) \xi(k)\right\} \\
& <-\sum_{i=1}^{r} \sum_{j=1}^{r} \sum_{l=1}^{r} h_{i, m} h_{j, m} h_{l, n} \psi^{T}(k) \Psi_{i j l, m} \psi(k) \\
& <-\sum_{l=1}^{r} h_{l, n} \psi^{T}(k) \Lambda^{T} \Psi_{l, m} \Lambda \psi(k)<0
\end{aligned}
$$

By considering the index $J$ in (23), together with (24)-(28), we obtain the following:

$$
J=E\left\{\sum_{k=0}^{\infty}\left[\Delta V(k)+z^{T}(k) z(k)-\gamma^{2} \xi^{T}(k) \xi(k)\right]\right\}<0
$$


Furthermore, under zero initial condition $\left(V\left(x(0), c_{0}\right)=0\right)$ and $E\left\{V_{\infty}\right\} \geq 0$, for $k=1,2, \ldots$, we have

$$
\begin{aligned}
& E\left\{\sum_{k=0}^{\infty} z^{T}(k) z(k)\right\}-\sum_{k=0}^{\infty} \gamma^{2} \xi^{T}(k) \xi(k) \\
& \quad<E\left\{V_{0}\right\}-E\left\{V_{\infty}\right\}<0
\end{aligned}
$$

It can be seen that (15) is a sufficient condition ensuring $J<0$. Next, (30) shows that the IT2FMJS is stochastically stable according to Definition 1. Thus, $\|z(k)\|_{E 2} \leqslant \gamma\|\xi(k)\|_{E 2}$ is proved to hold based on the above analysis.

Remark 1 In Theorem 1, the fuzzy-basis-dependent and mode-dependent Lyapunov function can reduce conservatism in stochastic stability.

\subsection{Controller Design}

Based on the analysis of Theorem 1, sufficient conditions on the existence of the desired state-feedback controller (12) will be given to ensure the closed-loop system (13) is stochastically stable with the optimized $H_{\infty}$ performance.

Theorem 2 By considering the closed-loop IT2FMJS (13) with incomplete transition probabilities and uncertain packet dropouts, for given $\gamma>0, \bar{\varepsilon}>0$, $\Delta \varepsilon, \sigma>0,|\Delta \varepsilon| \leq \sigma, m, n \in M, v \in M^{(m)}$, it is stochastically stable with a desired $H_{\infty}$ performance index $\gamma$ if there exist positive definite matrices $\left\{X_{i, m}\right\}$ $(i \in R, m \in M)$, matrices $\left\{N_{m}\right\}(m \in M),\left\{F_{i, m}\right\}(i \in R, m \in M)$, such that

$$
\left\{\begin{array}{l}
{\left[\begin{array}{ll}
\mathcal{X}_{i j l, m} & * \\
\mathcal{Y}_{i j l, m} & \mathcal{Z}_{i j l, m}
\end{array}\right]<0} \\
\Psi_{l, m}>0,(i \geq j, i, j, l \in R, m \in M)
\end{array}\right.
$$


where for $i \geqslant j, i, j, l \in R, m \in M, v \in M_{U K}^{(m)}$,

$$
\begin{aligned}
& \mathcal{X}_{i j l, m}=\left[\begin{array}{cc}
\mathcal{X}_{11} & * \\
\mathcal{X}_{21} & \mathcal{X}_{22}
\end{array}\right], \mathcal{Y}_{i j l, m}=\left[\mathcal{Y}_{11} \mathcal{Y}_{12}\right] \\
& \mathcal{Z}_{i j l, m}=\operatorname{diag}\left\{-\frac{1}{r} \widehat{I},-\widehat{X}_{l K_{1}}, \ldots,-\widehat{X}_{l K_{b}},-\widehat{X}_{l v}\right\} \\
& \mathcal{X}_{11}=-\frac{1}{r} \bar{X}+U_{i j l, m}, \mathcal{X}_{21}=V_{i j l, m} \\
& \mathcal{X}_{22}=-\frac{1}{r} \gamma^{2} I+W_{i j l, m}, \bar{X}=N_{m}^{T}+N_{m}-X_{i, m} \\
& \mathcal{Y}_{11}=\left[\bar{\Omega}_{i j, m}^{T} \bar{\Sigma}_{i j, m, 1}^{T} \sqrt{\rho_{U K}^{(m)}} \bar{\Phi}_{i j, m}^{T}\right]^{T}
\end{aligned}
$$

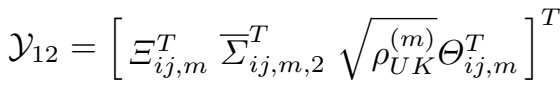

$$
\begin{aligned}
& \bar{\Sigma}_{i j, m, 1}=\left[\begin{array}{lll}
\sqrt{\rho_{m K_{1}}} & \bar{\Phi}_{i j, m}^{T} \cdots \sqrt{\rho_{m K_{b}}} \bar{\Phi}_{i j, m}^{T}
\end{array}\right]^{T} \\
& \bar{\Sigma}_{i j, m, 2}=\left[\sqrt{\rho_{m K_{1}}} \Theta_{i j, m}^{T} \cdots \sqrt{\rho_{m K_{b}}} \Theta_{i j, m}^{T}\right]^{T} \\
& \widehat{X}_{l, \vartheta}=\operatorname{diag}\left\{X_{l, \vartheta}, X_{l, \vartheta}\right\},\left(\vartheta \in M^{(m)}\right) \\
& \bar{\Omega}_{i j, m}=\Omega_{i j, m} N_{m}=\left[\begin{array}{l}
\theta_{1}\left(\widetilde{C}_{i, m}+\widetilde{C}_{j, m}\right) / 2 \\
\theta_{2} \widetilde{T}_{i j, m}
\end{array}\right] \\
& \widetilde{C}_{i, m}=C_{i, m} N_{m}, \widetilde{C}_{j, m}=C_{j, m} N_{m} \\
& \widetilde{T}_{i j, m}=\frac{1}{2}\left(\widetilde{C}_{i, m}+\widetilde{C}_{j, m}+D_{i, m} F_{j, m}+D_{j, m} F_{i, m}\right) \\
& F_{i, m}=K_{i, m} N_{m}, F_{j, m}=K_{j, m} N_{m} \\
& \bar{\Phi}_{i j, m}=\left[\begin{array}{c}
\theta_{1} \widetilde{\mathcal{D}}_{i j, m} \\
\theta_{2} \widetilde{S}_{i j, m}
\end{array}\right], \widetilde{\mathcal{D}}_{i j, m}=\frac{1}{2}\left(\widetilde{A}_{i, m}+\widetilde{A}_{j, m}\right) \\
& \widetilde{A}_{i, m}=A_{i, m} N_{m}, \widetilde{A}_{j, m}=A_{j, m} N_{m} \\
& \widetilde{S}_{i j, m}=\frac{1}{2}\left(\widetilde{A}_{i, m}+\widetilde{A}_{j, m}+B_{i, m} F_{j, m}+B_{j, m} F_{i, m}\right)
\end{aligned}
$$

The controller gains are given by

$$
K_{i, m}=F_{i, m} N_{m}^{-1},(i \in R, m \in M)
$$

Proof For any $i \in R$ and $m \in M$, according to $\bar{X}=N_{m}^{T}+N_{m}-X_{i, m}$, noting $X_{i, m}>0$, we have

$$
\begin{aligned}
& N_{m}^{T}+N_{m}-X_{i, m}-N_{m}^{T} X_{i, m}^{-1} N_{m} \\
& =-\left(X_{i, m}-N_{m}^{T}\right) X_{i, m}^{-1}\left(X_{i, m}-N_{m}\right) \leqslant 0
\end{aligned}
$$


According to (32) and (34), it is not difficult to obtain

$$
\overline{\mathcal{X}}_{11}=-\frac{1}{r} N_{m}^{T} X_{i, m}^{-1} N_{m}+U_{i j l, m} \leq \mathcal{X}_{11}
$$

Hence, applying (35) to (31) yields

$$
\left[\begin{array}{cc}
\overline{\mathcal{X}}_{i j l, m} & * \\
\mathcal{Y}_{i j l, m} & \mathcal{Z}_{i j l, m}
\end{array}\right]<0
$$

where

$$
\overline{\mathcal{X}}_{i j l, m}=\left[\begin{array}{cc}
\overline{\mathcal{X}}_{11} & * \\
\mathcal{X}_{21} & \mathcal{X}_{22}
\end{array}\right], \overline{\mathcal{X}}_{11}=-\frac{1}{r} N_{m}^{T} X_{i, m}^{-1} N_{m}+U_{i j l, m}
$$

By performing a congruence transformation to (31) by $\operatorname{diag}\left\{N_{m}^{-1}, I, I, \ldots, I\right\}$, the following can be obtained

$$
\left[\begin{array}{cc}
\widetilde{\mathcal{X}}_{i j l, m} & * \\
\widetilde{\mathcal{Y}}_{i j l, m} & \mathcal{Z}_{i j l, m}
\end{array}\right]<0
$$

where

$$
\begin{aligned}
& \tilde{\mathcal{X}}_{i j l, m}=\left[\begin{array}{cc}
\tilde{\mathcal{X}}_{11} & * \\
\widetilde{\mathcal{X}}_{21} & \mathcal{X}_{22}
\end{array}\right] \quad, \widetilde{\mathcal{Y}}_{i j l, m}=\left[\widetilde{\mathcal{Y}}_{11} \mathcal{Y}_{12}\right] \\
& \widetilde{\mathcal{X}}_{11}=-\frac{1}{r} X_{i, m}^{-1}+N_{m}^{-T} U_{i j l, m} N_{m}^{-1}, \widetilde{\mathcal{X}}_{21}=V_{i j l, m} N_{m}^{-1} \\
& \widetilde{\mathcal{Y}}_{11}=\left[\Omega_{i j, m}^{T} \Sigma_{i j, m, 1}^{T} \sqrt{\rho_{U K}^{(m)}} \Phi_{i j, m}^{T}\right]^{T}
\end{aligned}
$$

By defining $\widehat{P}_{l, m}=\widehat{X}_{l, m}^{-1}, \bar{U}_{i j l, m}=N_{m}^{-T} U_{i j l, m} N_{m}^{-1}, \bar{V}_{i j l, m}=V_{i j l, m} N_{m}^{-1}$, $\widehat{P}_{l, m}^{-1}=\operatorname{diag}\left\{P_{l, m}^{-1}, P_{l, m}^{-1}\right\}, i \geqslant j, i, j, l \in R, m \in M, v \in M_{U K}^{(m)}$, (37) can be rewritten as

$$
\left[\begin{array}{cc}
\mathcal{U}_{i j l, m} & * \\
\widetilde{\mathcal{Y}}_{i j l, m} & \mathcal{W}_{i j l, m}
\end{array}\right]<0
$$

where

$$
\begin{aligned}
& \mathcal{U}_{i j l, m}=\left[\begin{array}{cc}
-\frac{1}{r} P_{i, m}+\bar{U}_{i j l, m} & * \\
\bar{V}_{i j l, m} & -\frac{1}{r} \gamma^{2} I+W_{i j l, m}
\end{array}\right] \\
& \mathcal{W}_{i j l, m}=\operatorname{diag}\left\{\begin{array}{c}
1 \\
-\frac{1}{r} \bar{I},-\widehat{P}_{l, K_{1}}^{-1}, \ldots,-\widehat{P}_{l, K_{b}}^{-1},-\widehat{P}_{l, v}^{-1}
\end{array}\right\}
\end{aligned}
$$

By performing a congruence transformation to $\Psi_{l, m}$ in (31) by $\operatorname{diag}\left\{N_{m}^{-T}, I\right.$, $\left.\ldots, N_{m}^{-T}, I\right\}$, the following inequality can be obtained

$$
\bar{\Psi}_{l, m}=\left[\begin{array}{cccc}
\bar{\Psi}_{11 l, m} & * & * & * \\
\bar{\Psi}_{21 l, m} & \bar{\Psi}_{22 l, m} & * & * \\
\vdots & \vdots & \ddots & * \\
\bar{\Psi}_{r 1 l, m} & \bar{\Psi}_{2 r l, m} & \cdots & \bar{\Psi}_{r r l, m}
\end{array}\right]>0
$$


on the other hand, for any $i, j, l \in R, m \in M$, we have

$$
\bar{\Psi}_{i j l, m}=\left[\begin{array}{cc}
\bar{U}_{i j l, m} & * \\
\bar{V}_{i j l, m} & W_{i j l, m}
\end{array}\right]
$$

By Schur complement, (39) is rewritten as

$$
\mathcal{U}_{i j l, m}-\widetilde{\mathcal{Y}}_{i j l, m}^{T} \mathcal{W}_{i j l, m}^{-1} \widetilde{\mathcal{Y}}_{i j l, m}<0
$$

According to (32)-(39), (43) is expanded into

$$
\begin{aligned}
& {\left[\begin{array}{cc}
-\frac{1}{r} P_{i, m}+\bar{U}_{i j l, m} & * \\
\bar{V}_{i j l, m} & -\frac{1}{r} \gamma^{2} I+W_{i j l, m}
\end{array}\right]} \\
& \quad+\mathcal{O}_{i j, m}+\rho_{m K_{1}} \mathcal{Q}_{i j l, m K_{1}} \\
& \quad+\cdots+\rho_{m K_{b}} \mathcal{Q}_{i j l, m K_{b}}+\rho_{U K}^{(m)} \mathcal{Q}_{i j l, U K}<0
\end{aligned}
$$

where

$$
\begin{aligned}
& \mathcal{O}_{i j, m}=r \mathcal{J}_{i j, m}^{T} \widehat{I} \mathcal{J}_{i j, m}^{T}, \mathcal{J}_{i j, m}=\left[\Omega_{i j, m} \Xi_{i j, m}\right] \\
& \mathcal{Q}_{i j l, m K_{1}}=\mathcal{G}_{i j, m}^{T} \widehat{P}_{l, K_{1}} \mathcal{G}_{i j, m}, \mathcal{Q}_{i j l, m K_{b}}=\mathcal{G}_{i j, m}^{T} \widehat{P}_{l, K_{b}} \mathcal{G}_{i j, m} \\
& \mathcal{Q}_{i j l, U K}=\mathcal{G}_{i j, m}^{T} \widehat{P}_{l, v} \mathcal{G}_{i j, m}, \mathcal{G}_{i j, m}=\left[\Phi_{i j, m} \Theta_{i j, m}\right]
\end{aligned}
$$

According to (41) and (42), (44) can be written as

$$
\begin{aligned}
& {\left[\begin{array}{cc}
U_{i j l, m} & * \\
V_{i j l, m} & W_{i j l, m}
\end{array}\right]-\frac{1}{r}\left[\begin{array}{cc}
P_{i, m} & * \\
0 & \gamma^{2} I
\end{array}\right]} \\
& +r\left[\begin{array}{c}
\Omega_{i j, m}^{T} \\
\Xi_{i j, m}^{T}
\end{array}\right]\left[\Omega_{i j, m} \Xi_{i j, m}\right] \\
& +\left[\begin{array}{c}
\Phi_{i j, m}^{T} \\
\Theta_{i j, m}^{T}
\end{array}\right]\left(\sum_{\hbar=1}^{b} \rho_{m K_{\hbar}} \widehat{P}_{l, K_{\hbar}}\right)\left[\Phi_{i j, m} \Theta_{i j, m}\right] \\
& +\left[\begin{array}{c}
\Phi_{i j, m}^{T} \\
\Theta_{i j, m}^{T}
\end{array}\right]\left(\rho_{U K}^{(m)} \widehat{P}_{l, v}\right)\left[\Phi_{i j, m} \Theta_{i j, m}\right]<0
\end{aligned}
$$

Furthermore, according to Schur complement, (46) can be written as

$$
\begin{aligned}
& {\left[\begin{array}{cc}
\Phi_{i j, m}^{T} & \Omega_{i j, m}^{T} \\
\Theta_{i j, m}^{T} & \Xi_{i j, m}^{T}
\end{array}\right]\left[\begin{array}{cc}
\widehat{P} & * \\
0 & r \widehat{I}
\end{array}\right]\left[\begin{array}{ll}
\Phi_{i j, m} & \Theta_{i j, m} \\
\Omega_{i j, m} & \Xi_{i j, m}
\end{array}\right]} \\
& \quad-\frac{1}{r}\left[\begin{array}{cc}
P_{i, m} & * \\
0 & \gamma^{2} I
\end{array}\right]+\left[\begin{array}{ll}
U_{i j l, m} & * \\
V_{i j l, m} & W_{i j l, m}
\end{array}\right]<0
\end{aligned}
$$

where $\widehat{P}=\sum_{n \in M_{K}^{(m)}} \rho_{m n} \widehat{P}_{l, n}+\left(1-\rho_{K}^{(m)}\right) \widehat{P}_{l, v}$.

From (31)-(47), (47) is extrapolated from (31), i.e., (31) is a sufficient condition of (47). Hence, the developed criteria based on Theorem 2 are more conservative than that of Theorem 1. By comparing (47) and (15)-(17), it can 


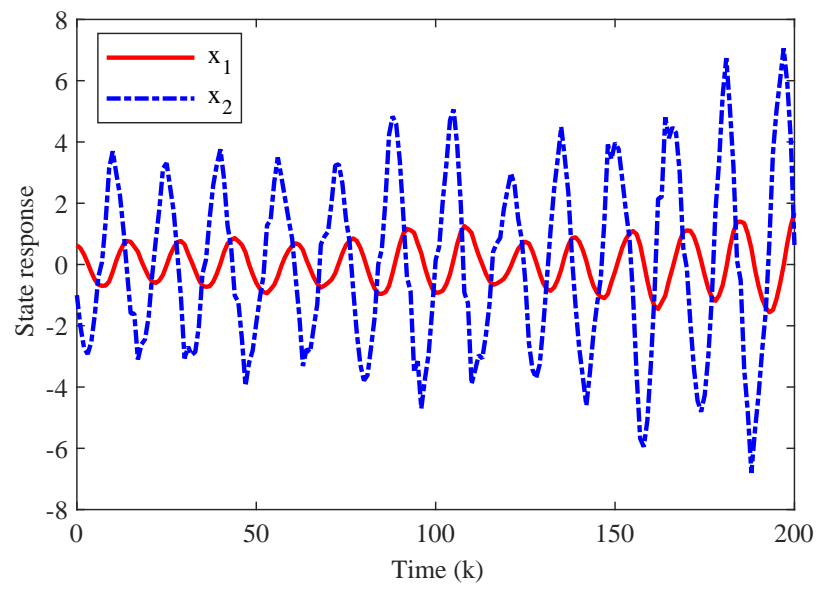

Fig. 1 State response of the open-loop system.

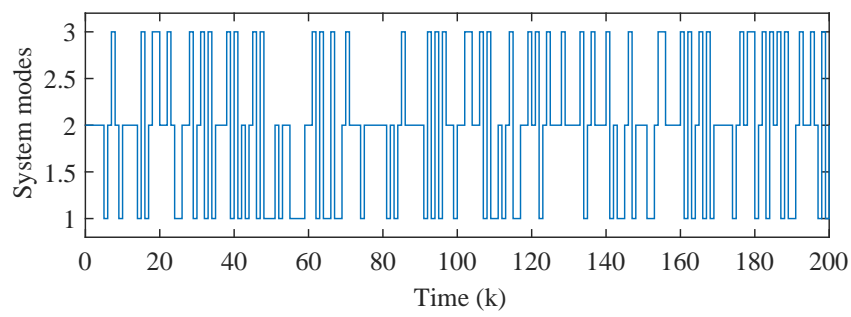

Fig. 2 Evolution of system mode.

be seen that (47) is a sufficient condition ensuring $\mathcal{A}_{i j, m}^{T} \mathcal{B A}_{i j, m}-\mathcal{C}_{i, m}+\Psi_{i j l, m}<$ 0 . Furthermore, in the mean square sense, stochastic stability of the closedloop system and the desired $H_{\infty}$ performance are ensured. This completes the proof.

Remark 2 It is observed that the calculation complexity of Theorem 2 depends on the number of unknown elements of TPM, system modes, and fuzzy rules. Thus, the number of unknown elements of TPM, the number of system modes, and the number of fuzzy rules should be appropriately chosen.

\section{An Illustrative Example}

In this section, a practical example is used to demonstrate the effectiveness and practicality of the proposed control approach.

\subsection{Application to a single-link robot arm system}

A single-link robotic arm system with Markov jump modes and uncertain packet dropouts used in $[21,33]$ is considered. The dynamic equation of the 


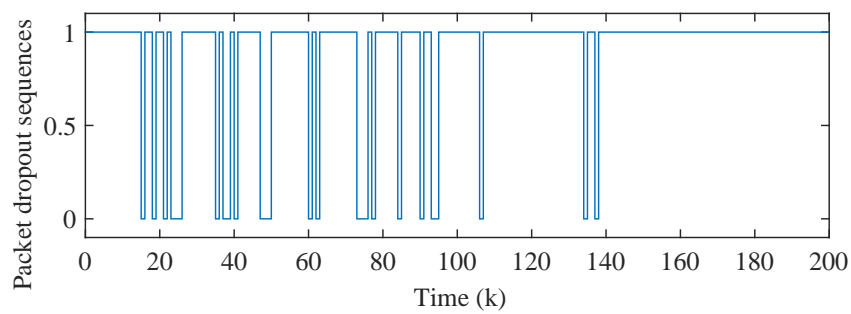

Fig. 3 Sequence of packet dropout .

robotic system is given by

$$
\ddot{\vartheta}(t)=-\frac{g L \mathcal{M}}{\mathcal{I}} \sin (\vartheta(t))-\frac{Q}{\mathcal{I}} \dot{\vartheta}(t)+\frac{1}{\mathcal{I}} u(t)+\xi(t)
$$

where $\theta(t), u(t)$, and $\xi(t)$ are the angle position of the robot arm, the control force, and the disturbance input belonging to $l_{2}[0, \infty)$, respectively. The terms $g, L$, and $\mathcal{M}$ denote gravitational acceleration, length of the arm, and mass of the load, respectively. The inertial moment and coefficient of viscous friction are represented by $\mathcal{I}$ and $Q$, respectively.

First, let $x_{1}(t)=\theta(t), x_{2}(t)=\dot{\theta}(t)$. In the system, two fuzzy rules and three system modes are set. The terms $\mathcal{M}$ and $\mathcal{I}$ have three values, i.e., $\mathcal{M}_{1}=1$, $\mathcal{M}_{2}=5, \mathcal{M}_{3}=10, \mathcal{I}_{1}=0.8, \mathcal{I}_{2}=1.2$, and $\mathcal{I}_{3}=1.6$ of which variations are subject to a Markov process. Besides, other parameters are set to $g=9.81$, $L=0.5$, and $Q=2$.

The sampling interval is set to $T=0.1$. Here, the fuzzy basis functions are designed by

$$
\left\{\begin{array}{l}
\lambda_{1}\left(x_{1}(k)\right)=\left\{\begin{array}{cc}
\frac{\sin \left(x_{1}(k)\right)-\eta x_{1}(k)}{(1-\eta) x_{1}(k)}, & x_{1}(k) \neq 0 \\
1 & x_{1}(k)=0
\end{array}\right. \\
\lambda_{2}\left(x_{1}(k)\right)=\left\{\begin{array}{cc}
\frac{x_{1}(k)-\sin \left(x_{1}(k)\right)}{(1-\eta) x_{1}(k)} & x_{1}(k) \neq 0 \\
0 & x_{1}(k)=0
\end{array}\right.
\end{array}\right.
$$

where $\eta$ denotes parameter uncertainties, $\eta \in[0.01,0.1] / \pi$. Hence, real fuzzy basis functions can be determined as follows:

$$
\left\{\begin{array}{l}
\lambda_{1}\left(x_{1}(k)\right)=\underline{\mu}_{1}\left(x_{1}(k)\right) \underline{\lambda}_{1}\left(x_{1}(k)\right)+\bar{\mu}_{1}\left(x_{1}(k)\right) \bar{\lambda}_{1}\left(x_{1}(k)\right) \\
\lambda_{2}\left(x_{1}(k)\right)=1-\lambda_{1}\left(x_{1}(k)\right)
\end{array}\right.
$$

where LUWCFs are denoted by $\underline{\mu}_{1}\left(x_{1}(k)\right)=\cos ^{2}\left(x_{1}(k)\right)$ and $\bar{\mu}_{1}\left(x_{1}(k)\right)=$ $1-\underline{\mu}_{1}\left(x_{1}(k)\right)$. It can be observed that when $x_{1}(k)$ is about $0 \mathrm{rad}, \lambda_{1}\left(x_{1}(k)\right)=1$ and $\lambda_{2}\left(x_{1}(k)\right)=0$; when $x_{1}(k)$ is about $\pm \pi \mathrm{rad}, \lambda_{1}\left(x_{1}(k)\right)=0$ and $\lambda_{2}\left(x_{1}(k)\right)=$ 1. Hence, the partition of fuzzy rules can be based on $x_{1}(k)$ with respect to 0 rad and $\pm \pi$ rad. 


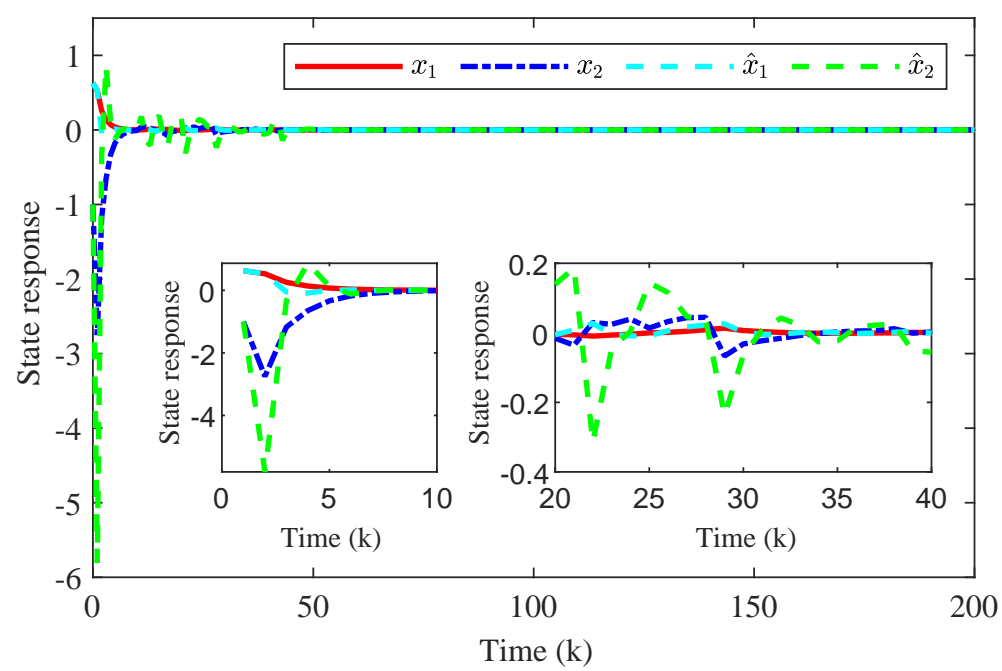

Fig. 4 State response of the closed-loop system with success data packet probability [0.9$0.1,0.9+0.1]$.

Next, the parameters of the IT2FMJS approximating the robot arm system are given by

$$
\begin{aligned}
& A_{1, s}=\left[\begin{array}{cc}
1 & T \\
-\frac{g L T M_{s}}{J_{s}} & 1-\frac{Q T}{J_{s}}
\end{array}\right], B_{i, s}=\left[\begin{array}{c}
0 \\
\frac{T}{J_{s}}
\end{array}\right] \\
& A_{2, s}=\left[\begin{array}{cc}
1 & T \\
-\frac{\eta g L T M_{s}}{J_{s}} & 1-\frac{Q T}{J_{s}}
\end{array}\right], G_{i, s}=\left[\begin{array}{c}
0 \\
T
\end{array}\right] \\
& C_{i, s}=\left[\begin{array}{ll}
1 & 0
\end{array}\right], D_{i, s}=0, H_{i, s}=0.1
\end{aligned}
$$

for $i=1,2,3$ and $s=1,2,3$. Besides, the TPM with partly unknown elements is given by

$$
\Upsilon=\left[\begin{array}{ccc}
? & 0.5 & ? \\
0.2 & 0.5 & 0.3 \\
0.6 & ? & ?
\end{array}\right]
$$

where "?" denotes an unknown element.

Fig. 1 shows the state response of the open-loop system $(u(k) \equiv 0$ in (13)). Evolution of the system mode under the condition of (50) and the packet dropout sequence with nominal expectation $\bar{\varepsilon}$ and uncertainty $\sigma$ are shown in Figs. 2 and 3, respectively. It can be seen from Fig. 1 that the open-loop system is unstable. Comparative studies of optimal $H_{\infty}$ performance indices $\gamma_{\min }$ for different packet dropout cases under the condition of (50) are given in Table I. As $\varepsilon$ increases, the optimal $H_{\infty}$ performance index $\gamma_{\min }$ becomes smaller, which means that more reliable communication conditions and smaller value of $\gamma_{\min }$ lead to better $H_{\infty}$ performance. Furthermore, for a fixed $\varepsilon$, Tablē I shows 
Table 1 Optimal $H_{\infty}$ Performance Indices $\gamma_{\min }$ for Different $\bar{\varepsilon}$ and $\sigma$ Under the Condition of $(50)$

\begin{tabular}{c||ccccc}
\hline $\bar{\varepsilon}$ & 0.7 & 0.75 & 0.8 & 0.85 & 0.9 \\
\hline$\sigma=0$ & infeasible & 1.3153 & 0.3760 & 0.2789 & 0.2486 \\
$\sigma=0.02$ & infeasible & infeasible & 0.5732 & 0.3135 & 0.2650 \\
$\sigma=0.04$ & infeasible & infeasible & 2.1471 & 0.3864 & 0.2870 \\
$\sigma=0.06$ & infeasible & infeasible & infeasible & 0.6257 & 0.3200 \\
$\sigma=0.08$ & infeasible & infeasible & infeasible & infeasible & 0.3923 \\
$\sigma=0.10$ & infeasible & infeasible & infeasible & infeasible & 0.6662 \\
\hline
\end{tabular}

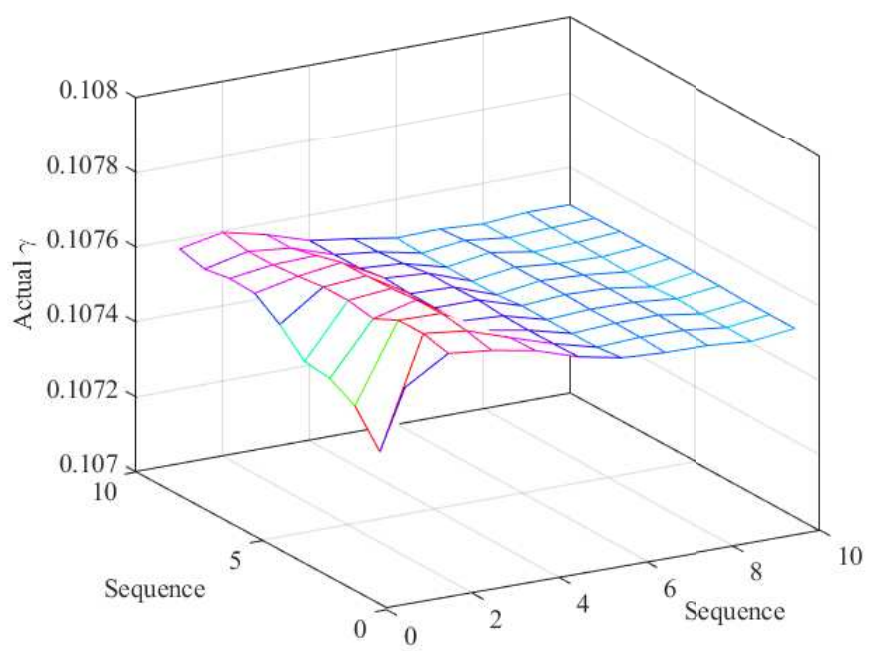

Fig. 5 Actual $H_{\infty}$ performance indices $\gamma$ for 100 different Markov jump sequences.

that $\gamma_{\min }$ increases as $\sigma$ grows, which makes the $H_{\infty}$ performance worse. This illustrates that nominal expectation and uncertainties of the packet dropout have great effects on the desired performance. The initial state values are set to $x_{0}=[0.25 \pi-1]$. To test $H_{\infty}$ performance, zero initial condition with disturbance input are assumed, where

$$
\xi(k)=\left\{\begin{array}{cc}
e^{-0.1(k-10)} \sin (k-10), 1 \leq k \leq 50 \\
0, & \text { else }
\end{array}\right.
$$

When $\bar{\varepsilon}=0.9$ and $\sigma=0.1$, the closed-loop state responses of $x_{1}$ and $x_{2}$ converge to zero, as shown in Fig. 4, which shows that the designed controller is efficient against incomplete transition probability and uncertain packet dropout.

To evaluate the $H_{\infty}$ performance, actual $H_{\infty}$ performance indices computed by $\sqrt{\sum_{h=0}^{k} z(h)^{T} z(h)} / \sqrt{\sum_{h=0}^{k} \xi(h)^{T} \xi(h)}$ under zero-initial condition, and $\bar{\varepsilon}=0.9, \sigma=0.1$ with $\Upsilon$ are shown in Fig. 5 . It is observed that values of actual $H_{\infty}$ performance indices $\gamma$ for 100 different Markov jump sequences 
under the same condition are almost identical and are all smaller than the optimal value of $\gamma_{\min }=0.6662$ in Table I. This shows that variations of random sequences have little effect on the system performance. Besides, actual $H_{\infty}$ performance indices about two packet dropout sequences corresponding to $\sigma=0.1$ and $\sigma=0$ are $\gamma_{1}=0.1078$ and $\gamma_{2}=0.1064$, respectively. By employing Theorem 2 , the controller gains $\mathcal{K}(0.9,0.1)$ and $\mathcal{K}(0.9,0)$ are obtained for $\sigma=0.1$ and $\sigma=0$, respectively. If the controller gain $\mathcal{K}(0.9,0)(\sigma=0)$ is adopted to test the packet dropout sequence with uncertainty $\sigma=0.1$, actual $H_{\infty}$ performance indices can be calculated as $\gamma_{3}=0.1075$ and the resulting state responses of $\widehat{x}_{1}$ and $\widehat{x}_{2}$ are obtained in Fig. 4 . It can be seen from $\gamma_{3}>\gamma_{2}$ that uncertainties of packet dropout need to be considered in the controller design. Simultaneously, by comparing $x_{1}, x_{2}$ with $\widehat{x}_{1}, \widehat{x}_{2}$, the controller with uncertain packet dropout is much better than that without the uncertain packet dropout in terms of overshoot and convergence time.

To investigate the effect of incomplete TPM on $H_{\infty}$ performance of the system, values of known elements in the rows with unknown elements will be increased, i.e., sum of unknown elements in the same rows will be reduced. Compared with (50), the TPM after enlarging values of known elements is given by

$$
\widetilde{\Upsilon}=\left[\begin{array}{ccc}
? & \mathbf{0 . 6} & ? \\
0.2 & 0.5 & 0.3 \\
\mathbf{0 . 7} & ? & ?
\end{array}\right]
$$

where "?" denotes unknown element and boldface denotes enlarged element.

Under the condition of (52), comparison of optimal $H_{\infty}$ performance indices $\gamma_{\min }$ for different $\bar{\varepsilon}$ with different $\sigma$ is obtained in Table II. By comparing Table I with Table II, $\widetilde{\gamma}_{\text {min }}$ with $\widetilde{\Upsilon}$ is smaller than $\gamma_{\text {min }}$ with $\Upsilon$ when $\bar{\varepsilon}$ ranges from 0.7 to 0.9 and $\sigma$ ranges from 0 to 0.1 . This indicates that the increase of known degree of description information in the TPM will improve the system performance.

To further test the effect of the known degree of description information in the TPM on the $H_{\infty}$ performance, let $\rho_{12}$ and $\rho_{31}$ in $\Upsilon$ and $\widetilde{\Upsilon}$ vary from 0.2 from 0.8 to test minimum $H_{\infty}$ performance indices $\gamma_{\min }$, as shown in Fig. 6 . The main trend of $\gamma_{\min }$ is decreasing with increase of $\rho_{12}$ and $\rho_{31}$. It can seen that the more complete the description information of the TPM is, the better $H_{\infty}$ performance is with smaller the value of $\gamma_{\min }$.

\section{Conclusion}

This paper has addressed fuzzy $H_{\infty}$ control problem for a class of discrete-time IT2FMJS with incomplete transition probabilities under unreliable communication links. A more practical scenario that the transition probability is incomplete and the packet dropout is uncertain is considered. A mode-dependent and fuzzy-basis-dependent Lyapunov function is presented to obtain a less conservative stochastic stability condition. Based on the above dependent function 


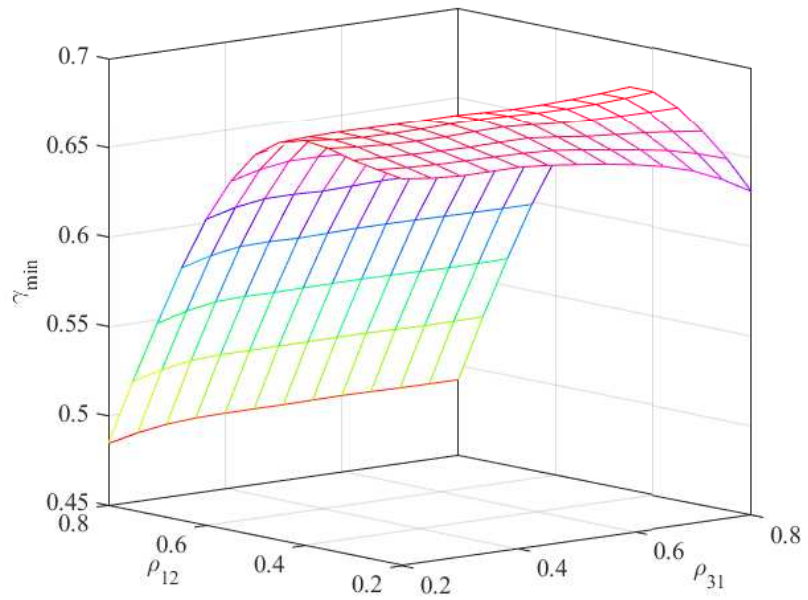

Fig. 6 Minimum $H_{\infty}$ performance indices $\gamma_{\min }$ for different incomplete TPMs.

Table 2 Optimal $H_{\infty}$ Performance Indexes $\gamma_{\min }$ for Different $\bar{\varepsilon}$ and $\sigma$ Under the Condition of (50)

\begin{tabular}{c||ccccc}
\hline $\bar{\varepsilon}$ & 0.7 & 0.75 & 0.8 & 0.85 & 0.9 \\
\hline$\sigma=0$ & infeasible & 1.1531 & 0.3701 & 0.2781 & 0.2483 \\
$\sigma=0.02$ & infeasible & infeasible & 0.5473 & 0.3114 & 0.2647 \\
$\sigma=0.04$ & infeasible & infeasible & 1.6970 & 0.3800 & 0.2860 \\
$\sigma=0.06$ & infeasible & infeasible & infeasible & 0.5928 & 0.3185 \\
$\sigma=0.08$ & infeasible & infeasible & infeasible & infeasible & 0.3858 \\
$\sigma=0.10$ & infeasible & infeasible & infeasible & infeasible & 0.6281 \\
\hline
\end{tabular}

method, a fuzzy $H_{\infty}$ controller has been proposed to guarantee that the closedloop IT2FMJS is stochastically stable with a desired performance index. The proposed approach overcomes two important practical problems, which can be also applied to address filtering and state estimation problems for the resulting system.

\section{Acknowledgments}

This work was supported in part by the China Scholarship Council under Grant (No. 201906120072).

Conflict of interest. The authors declare that they have no conflict of interest concerning the publication of this manuscript.

\section{References}

1. Boukas, E.K., Liu, Z.K.: Roubust $H_{\infty}$ control of discrete-time markovian jump linear systems with mode-dependent time-delays. IEEE Trans. Autom. Control 46(12), 1918$1924(2001)$ 
2. Cheng, J., Huang, W., Park, J.H., Cao, J.: A hierarchical structure approach to finitetime filter design for fuzzy markov switching systems with deception attacks. IEEE Transactions on Cybernetics (10.1109/TCYB.2021.3049476)

3. Cheng, J., Park, J.H., Wang, H.: Event-triggered $H_{\infty}$ control for T-S fuzzy nonlinear systems and its application to truck-trailer system. ISA Trans. 65, 62-71 (2016)

4. Dong, S., Wu, Z., Shi, P., Su, H., Huang, T.: Quantized control of markov jump nonlinear systems based on fuzzy hidden markov model. IEEE Trans. Cybern. 49(7), 2420-2430 (2019)

5. Elahi, A., Alfi, A., Modares, H.: $H_{\infty}$ consensus of homogeneous vehicular platooning systems with packet dropout and communication delay. IEEE Trans. Syst., Man, Cybern., Syst. (10.1109/TSMC.2021.3071994)

6. Guo, X., Fan, X., Ahn, C.K.: Adaptive event-triggered fault detection for interval type-2 T-S fuzzy systems with sensor saturation. IEEE Trans. Fuzzy Syst. (DOI: 10.1109/TFUZZ.2020.2997515)

7. $\mathrm{Hu}, \mathrm{Z} ., \mathrm{Mu}, \mathrm{X}$.: Mean square stabilization for sampled-data ts fuzzy systems with random packet dropout. IEEE Trans. Fuzzy Syst. 28(8), 1815-1824 (2020)

8. Kim, S.H.: Dissipative control of markovian jump fuzzy systems under nonhomogeneity and asynchronism. Nonlinear Dyn. 97, 629-646 (2019)

9. Kwon, N.K., Park, I.S., Park, P.: $\mathcal{H}_{\infty}$ state-feedback control for continuous-time markovian jump fuzzy systems using a fuzzy weighting-dependent lyapunov function. Nonlinear Dyn. 90, 2001-2011 (2017)

10. Lam, H.K., Li, H., Deters, C., Secco, E.L., Wurdemann, H.A., Althoefer, K.: Control design for interval type-2 fuzzy systems under imperfect premise matching. IEEE Trans. Ind. Electron. 61(2), 956-968 (2014)

11. Lam, H.K., Tsai, S.H.: Stability analysis of polynomial-fuzzy-model-based control systems with mismatched premise membership functions. IEEE Trans. Fuzzy Syst. 22(1), 223-229 (2014)

12. Li, H., Yin, S., Pan, Y., Lam, H.K.: Model reduction for interval type-2 takagisugeno fuzzy systems. Automatica 61, 308-314 (2015)

13. Li, L., Shen, M., Zhang, G., Yan, S.: $H_{\infty}$ control of markov jump systems with timevarying delay and incomplete transition probabilities. Applied Mathematics and Computation 301, 95-106 (2017)

14. Li, M., Chen, Y.: A wide-area dynamic damping controller based on robust $H_{\infty}$ control for wide-area power systems with random delay and packet dropout. IEEE Trans. Power Syst. 33(4), 4026-4037 (2018)

15. Liu, J., Ran, G., Huang, Y., Han, C., Yu, Y., Sun, C.: Adaptive event-triggered finitetime dissipative filtering for interval type-2 fuzzy markov jump systems with asynchronous modes. IEEE Trans. Cybern. (10.1109/TCYB.2021.3053627)

16. Liu, J., Wu, C., Wang, Z., Wu, L.: Reliable filter design for sensor networks using type-2 fuzzy framework. IEEE Trans. Ind. Inform. 13(4), 1742-1752 (2017)

17. Nguyen, T.B., Kim, S.H.: Dissipative control of interval type-2 nonhomogeneous markovian jump fuzzy systems with incomplete transition descriptions. Nonlinear Dyn. 100, 1289-1308 (2020)

18. Ning, Z., Cai, B., Weng, R., Zhang, L.: Nonsynchronized state estimation for fuzzy markov jump affine systems with switching region partitions. IEEE Trans. Cybern. (DOI: 10.1109/TCYB.2020.3002938)

19. Qi, W., Yang, X., Gao, X., Cheng, J., Kao, Y., Wei, Y.: Stability for delayed switched systems with markov jump parameters and generally incomplete transition rates. Applied Mathematics and Computation 365, 124718 (2020)

20. Shan, Y., Liu, X., She, K., Zhong, S., Cheng, J., Zhang, X.: Extended dissipative asynchronous filtering for $\mathrm{T}-\mathrm{S}$ fuzzy switched systems with partial transition descriptions and incomplete measurements. Nonlin. Anal.: Hybrid Syst. 37, 100906 (2020)

21. Shi, S., Fei, Z., Shi, P., Ahn, C.K.: Asynchronous filtering for discrete-time switched T-S fuzzy systems. IEEE Trans. Fuzzy Syst. 28(8), 1531-1541 (2020)

22. Song, X., Wang, M., Ahn, C.K., Song, S.: Finite-time $H_{\infty}$ asynchronous control for nonlinear markov jump distributed parameter systems via quantized fuzzy output-feedback approach. IEEE Trans. Cybern. 50(9), 4098-4109 (2020) 
23. Sun, D., Liao, Q., Ren, H.: Type-2 fuzzy modeling and control for bilateral teleoperation system with dynamic uncertainties and time-varying delays. IEEE Trans. Ind. Electron. 65(1), 447-459 (2018)

24. Tan, C., Li, L., Zhang, H.: Stabilization of networked control systems with both networkinduced delay and packet dropout. Automatica 59, 194-199 (2015)

25. Tanaka, K., Wang, H.O.: Fuzzy Control Systems Design and Analysis: A Linear Matrix Inequality Approach. Wiley, New York (2001)

26. Tao, J., Lu, R., Su, H., Shi, P., Wu, Z.: Asynchronous filtering of nonlinear markov jump systems with randomly occurred quantization via $\mathrm{T}-\mathrm{S}$ fuzzy models. IEEE Trans. Fuzzy Syst. 26(4), 1866-1877 (2018)

27. Wu, Z., Dong, S., Su, H., Li, C.: Asynchronous dissipative control for fuzzy markov jump systems. IEEE Trans. Cybern. 48(8), 2426-2436 (2018)

28. Xing, M., Wang, Y., Zhuang, G., Zhang, M.: Dynamic event-based dissipative asynchronous control for ts fuzzy singular markov jump lpv systems against deception attacks. Nonlinear Dyn. 103, 1709-1731 (2021)

29. Xu, Z., Xi, B., Yi, G., Wang, D.: A novel model for fully closed-loop system of hemispherical resonator gyroscopes under force-to-rebalance mode. IEEE Trans. Instrumen. Meas. 69(12), 9918-9930 (2020)

30. Xu, Z., Zhu, W., Yi, G., Fan, W.: Dynamic modeling and output error analysis of an imperfect hemispherical shell resonator. J. Sound Vibr. 498, 115964 (2021)

31. Zhang, L., Boukas, E., Lam, J.: Analysis and synthesis of markov jump linear systems with time-varying delays and partially known transition probabilities. IEEE Trans. Autom. Control 53(10), 2458-2464 (2008)

32. Zhang, L., Lam, J.: Necessary and sufficient conditions for analysis and synthesis of markov jump linear systems with incomplete transition description. IEEE Trans. Autom. Control 55(7), 1695-1701 (2010)

33. Zhang, L., Ning, Z., Shi, P.: Input-output approach to control for fuzzy markov jump systems with time-varying delays and uncertain packet dropout rate. IEEE Trans. Cybern. 45(11), 2449-2460 (2015)

34. Zhang, M., Shi, P., Ma, L., Cai, J., Su, H.: Quantized feedback control of fuzzy markov jump systems. IEEE Trans. Cybern. 49(9), 3375-3384 (2019)

35. Zhang, Y., Xu, S., Zhang, B.: Robust output feedback stabilization for uncertain discrete-time fuzzy markovian jump systems with time-varying delays. IEEE Trans. Fuzzy Syst. 17(2), 411-420 (2009)

36. Zhang, Z., Shi, Y., Zhang, Z., Yan, W.: New results on sliding-mode control for TakagiSugeno fuzzy multiagent systems. IEEE Trans. Cybern. 49(5), 1592-1604 (2019) 
Figures

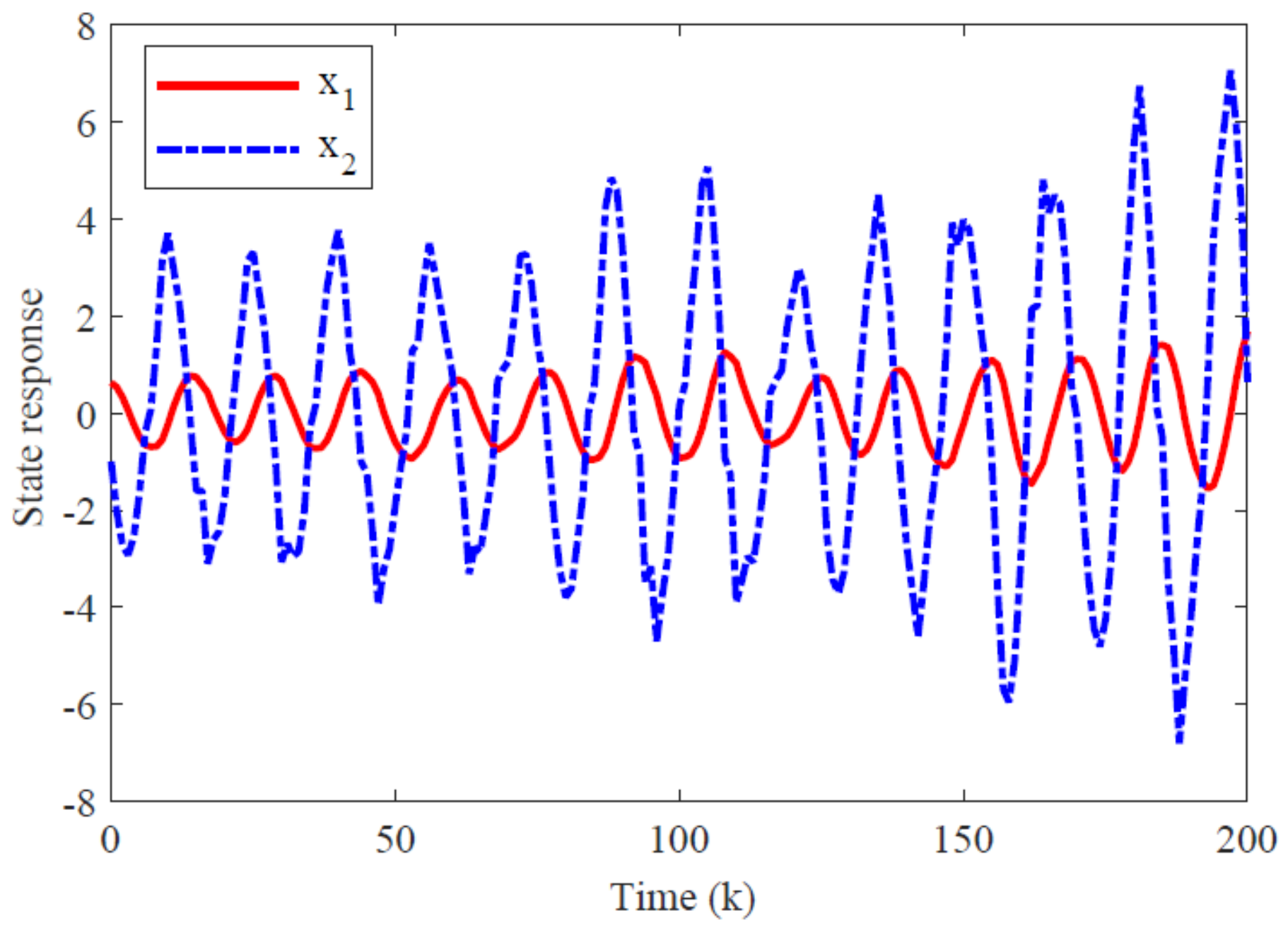

Figure 1

State response of the open-loop system.

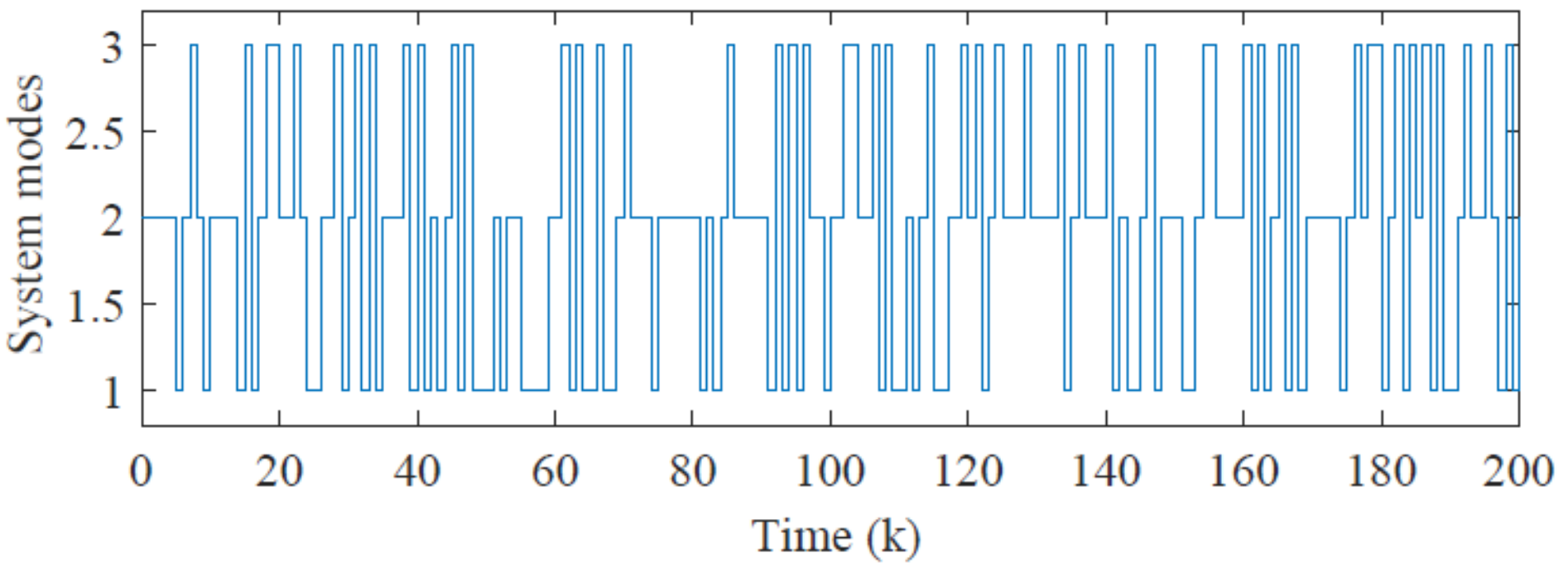

Figure 2 
Evolution of system mode.

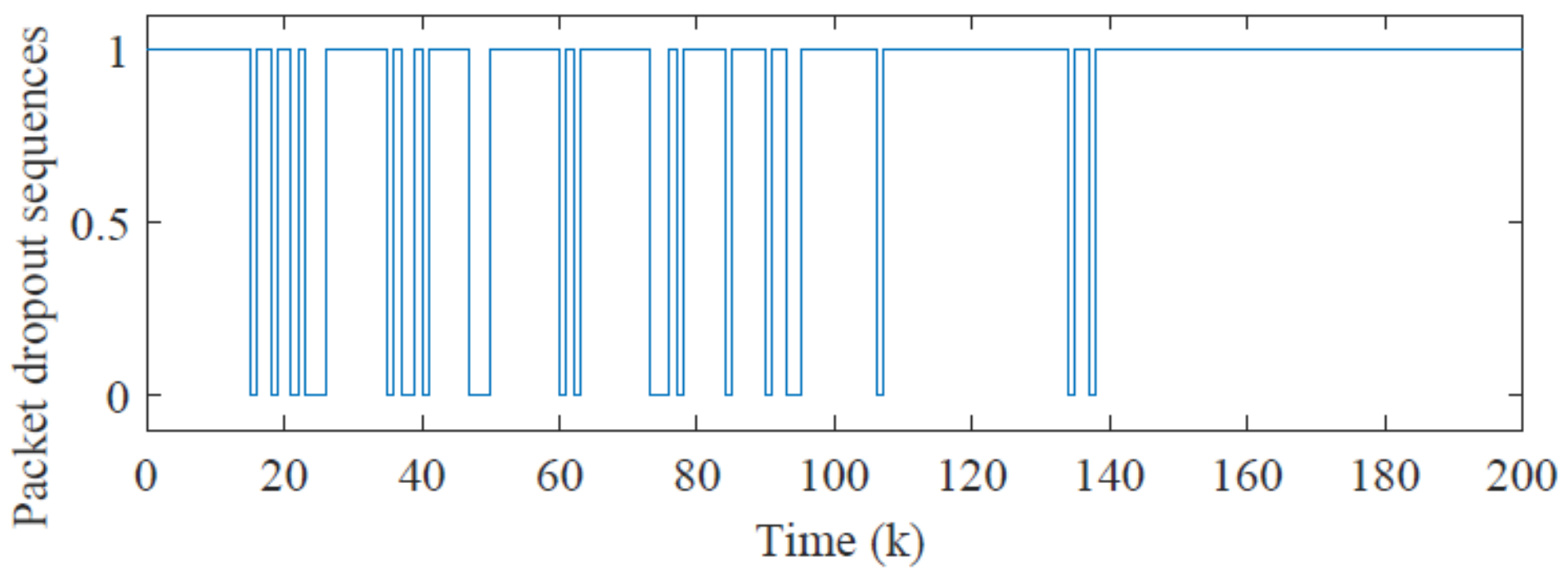

Figure 3

Sequence of packet dropout.

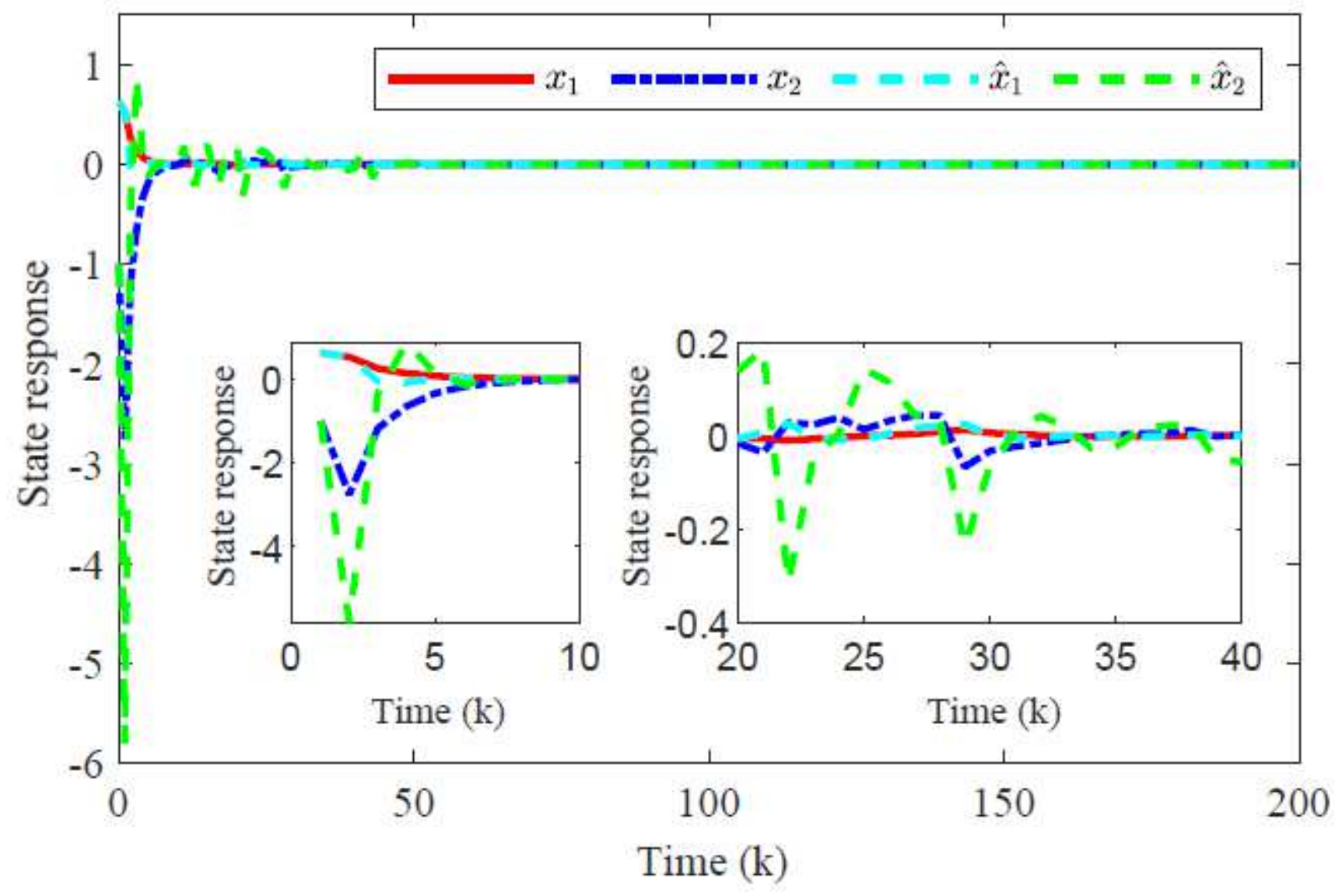

Figure 4

State response of the closed-loop system with success data packet probability $[0.9-0.1,0.9+0.1]$. 


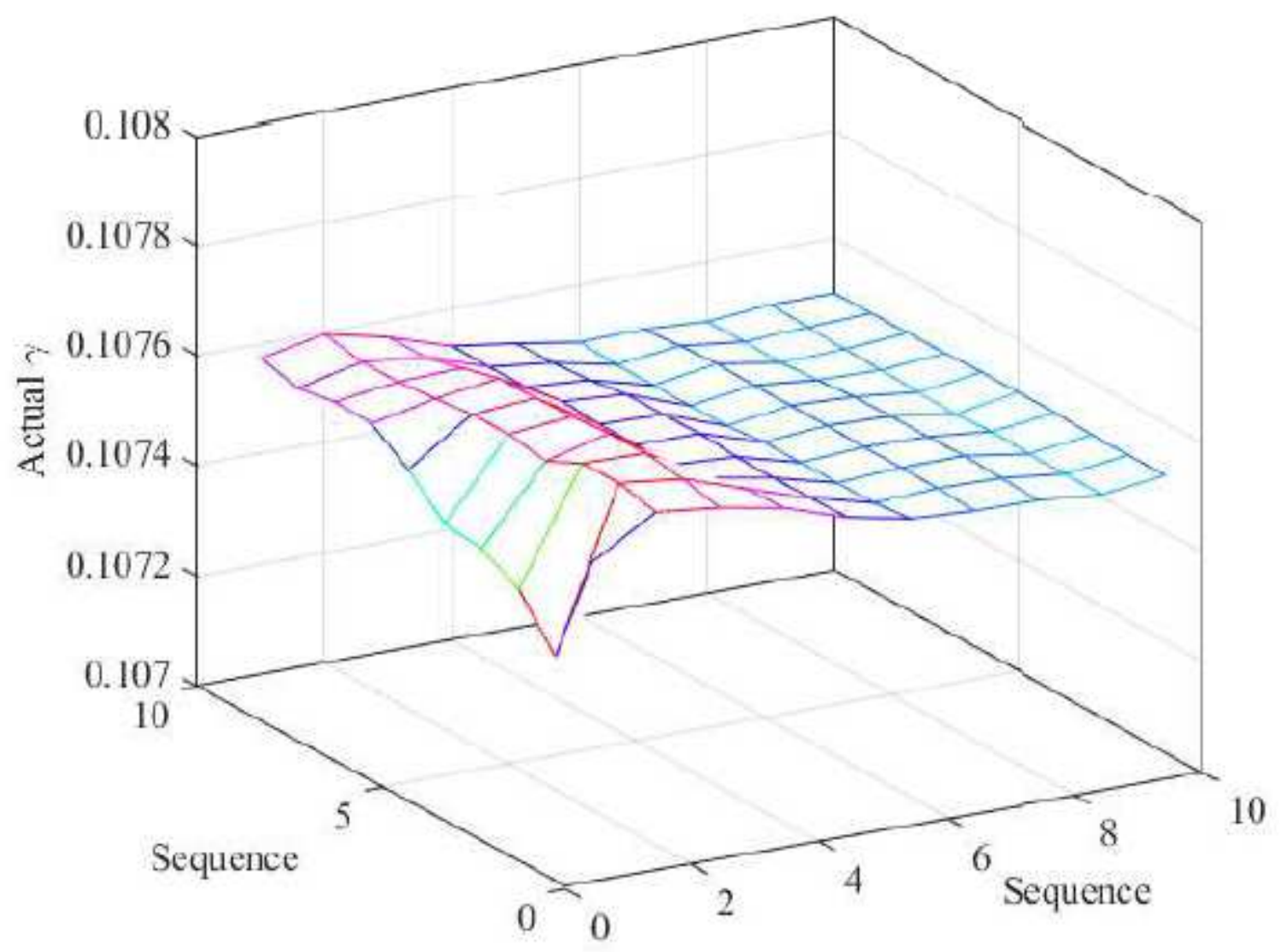

Figure 5

Actual $\mathrm{H} \infty$ performance indices for 100 different Markov jump sequences.

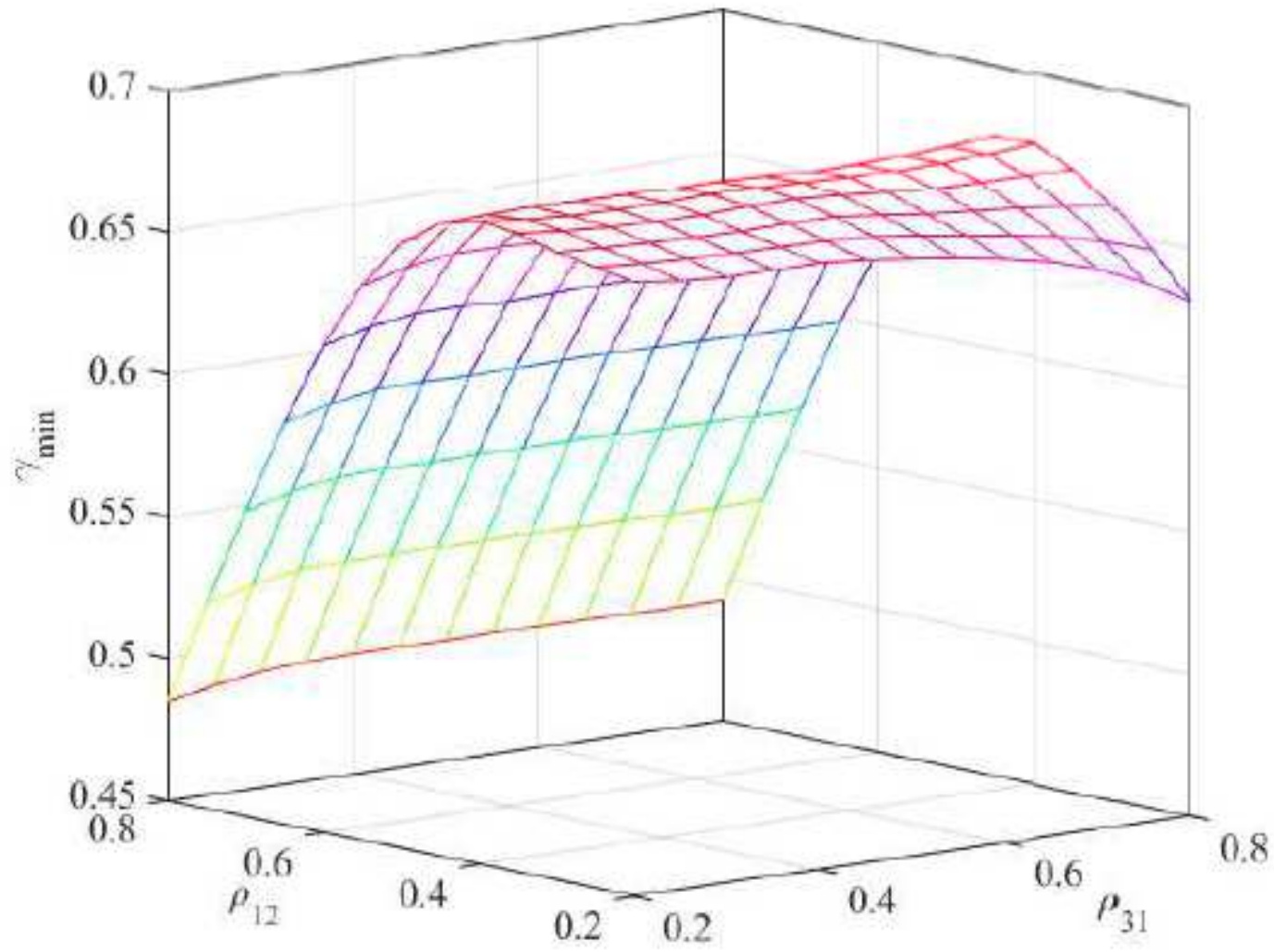


Figure 6

Minimum $\mathrm{H}^{\infty}$ performance indices min for different incomplete TPMs. 